\title{
3D controlled-source electromagnetic modeling in anisotropic medium using edge-based finite element method
}

\author{
Hongzhu Cai ${ }^{\mathrm{a}}$, Bin Xiong ${ }^{\mathrm{b}, *}$, Muran Han ${ }^{\mathrm{a}}$, Michael Zhdanov ${ }^{\mathrm{a}, \mathrm{c}, \mathrm{d}}$ \\ ${ }^{a}$ Consortium for Electromagnetic Modeling and Inversion (CEMI), University of Utah, Salt Lake City, UT 84112, USA \\ ${ }^{\mathrm{b}}$ College of Earth Sciences, Guilin University of Technology, Guilin, Guangxi 541004, China \\ c TechnoImaging, Salt Lake City, UT 84107, USA \\ ${ }^{\mathrm{d}}$ Moscow Institute of Physics and Technology, Moscow 141700, Russia
}

\section{A R T I C L E I N F O}

Available online 5 October 2014

Keywords:

Numerical solutions

Marine electromagnetics

Electromagnetic theory

Electrical anisotropy

Finite element

\begin{abstract}
A B S T R A C T
This paper presents a linear edge-based finite element method for numerical modeling of 3D controlledsource electromagnetic data in an anisotropic conductive medium. We use a nonuniform rectangular mesh in order to capture the rapid change of diffusive electromagnetic field within the regions of anomalous conductivity and close to the location of the source. In order to avoid the source singularity, we solve Maxwell's equation with respect to anomalous electric field. The nonuniform rectangular mesh can be transformed to hexahedral mesh in order to simulate the bathymetry effect. The sparse system of finite element equations is solved using a quasi-minimum residual method with a Jacobian preconditioner. We have applied the developed algorithm to compute a typical MCSEM response over a 3D model of a hydrocarbon reservoir located in both isotropic and anisotropic mediums. The modeling results are in a good agreement with the solutions obtained by the integral equation method.
\end{abstract}

(c) 2014 Elsevier Ltd. All rights reserved.

\section{Introduction}

Controlled-source electromagnetic (CSEM) method has been widely used in geophysical exploration on land for decades (Ward and Hohmann, 1988; Zhdanov and Keller, 1994). The marine controlled-source electromagnetic (MCSEM) method was also applied for the off-shore hydrocarbon (HC) exploration (Srnka et al., 2006; Constable and Srnka, 2007; Um and Alumbaugh, 2007; Andréis and MacGregor, 2008; Zhdanov, 2010). The subsurface conductivity structure could be very complex due to bathymetry and a lateral variation of the conductivity of the sea-bottom sediments. In this case, a full 3D modeling of diffusive electromagnetic data is desirable to correctly interpret the field MCSEM data (Silva et al., 2012). The 3D electromagnetic modeling requires solving the diffusive Maxwell's equations in a discretized form. The most popular numerical techniques for EM forward modeling are integral equation (IE), finite difference (FD), and finite element (FE) methods.

Compared to the integral equation and finite difference methods, the finite element method is more suitable for modeling of EM response in a complex geoelectrical structure. In a 3D scenario,

\footnotetext{
* Corresponding author

E-mail addresses: caihongzhu@hotmail.com (H. Cai), xiongbin@msn.com (B. Xiong), michael.s.zhdanov@gmail.com (M. Zhdanov).
}

the subsurface can be discretized using either regular brick, hexahedral, or tetrahedron elements. The electric and magnetic fields within each element can be approximated by either linear or higher order polynomial functions. Since the support of the finite element basis function is small, the resulting stiffness matrix is very sparse, which makes it easy to store. In the paper, we also compared the sparsity pattern of the stiffness matrix created by our finite element method and that from the finite difference method. Although the finite element stiffness matrix is less sparse than the finite difference stiffness matrix for the same model, the bandwidth of finite element stiffness matrix is much narrower.

The node-based finite element method was applied in the past to model EM data by solving the coupled equations for the vector and scalar potentials and also by solving Maxwell's equations for electric and magnetic fields (e.g., Zhdanov, 2009). However, for accurate computations, the divergence free condition for the electric and magnetic fields in the source free regions needs to be addressed by an additional penalty term to alleviate possible spurious solutions (e.g., Jin, 2002).

The advantage of the edge-based finite element method, introduced by Nédélec (1980), is that the divergence free conditions are satisfied automatically by an appropriate selection of the basis functions. The basis function of the Nédélec element is a vector function defined along the element edges and at the center of each edge. The tangential continuity of either electric or magnetic field is imposed automatically on the element's 
interfaces while the normal components are still can be discontinuous (Jin, 2002). In this paper, we present the formulation of Maxwell's equation for the electric field directly using edge-based finite element approach and the continuity of tangential electric field can be imposed directly. We can also formulate Maxwell's equation for magnetic field in the same way and the continuity of tangential magnetic field will be imposed directly in the formulation. In spite of the fact that the edge-based finite element method was widely used in electrical engineering for over 30 years, it started gaining the interest from the geophysical community recently only. Mitsuhata and Uchida (2004) implemented an edge-based finite element modeling algorithm for solving 3D magnetotelluric problem. Schwarzbach et al. (2011) applied linear and higher order edge element for the modeling of marine CSEM data using tetrahedron discretization to better simulate the seafloor bathymetry. Silva et al. (2012) proposed a finite element multifrontal method which is very efficient for 3D CSEM modeling in the frequency domain. One needs to note that all these formulations of the 3D CSEM problem assume the subsurface conductivity to be isotropic.

In the marine environment, the subsurface conductivity is usually characterized by strong anisotropy due to sedimentation. Generally the subsurface is more conductive in the horizontal direction than in the vertical direction for a horizontally stratified medium. The anisotropy of conductive sediment can affect the response of the electromagnetic field in a marine CSEM survey and this effect has already been well studied (Ramananjaona et al., 2011; Ellis et al., 2010; Brown et al., 2012; Newman et al., 2010). Obviously, to accurately interpret the marine CSEM data, the conductivity anisotropy needs to be considered in the forward modeling. There are already series of papers published on $2.5 \mathrm{D}$ and 3D modeling of marine CSEM data in the anisotropic medium using node-based finite element and finite difference methods (Kong et al., 2008; Weiss and Newman, 2002).

Meanwhile, another challenge arising in the interpretation of MCSEM data is strong distortion of the data by the effect of seafloor bathymetry (e.g., Sasaki, 2011). For accurate interpretation of the subsurface structure using the MCSEM method, the bathymetry effect should be accurately simulated. The finite element method is very well suited to solve this problem.

In this paper, we formulate the 3D marine CSEM problem using the linear edge-element method in the anisotropic medium. In a general case, we assume that the model has a triaxial conductivity anisotropy. In order to compare the EM response with integral equation solution (Zhdanov et al., 2006), we also consider transverse anisotropy in our model study. To avoid the source singularity, we solve Maxwell's equations with respect to anomalous electric field. The background EM field for the layered background model is computed using Hankel transforms (Anderson, 1989; Guptasarma and Singh, 1997). For simplicity, we use a rectangular element for the flat seafloor model. In order to simulate the bathymetry effect, the rectangular element is transformed into hexahedral one by shifting the vertical coordinate. The sparse finite element system of equations is solved using a quasi-minimum residual method (QMR) with a Jacobian preconditioner.

To validate our code, we first test it for a 1D model with an analytical solution. For a full 3D anisotropic problem, we compare the numerical results from our method and integral equation solutions.

\section{Formulation of the EM field equations with respect to anomalous field in anisotropic medium}

The low frequency electromagnetic field, considered in geophysical application, satisfies the following Maxwell's equations
(Zhdanov, 2009):

$\nabla \times \mathbf{E}=i \omega \mu_{0} \mathbf{H}$

$\nabla \times \mathbf{H}=\mathbf{J}_{S}+\bar{\sigma} \mathbf{E}$

where we adopt the harmonic time dependence $e^{-i \omega t}, \omega$ is the angular frequency, $\mu_{0}$ is the free space magnetic permeability, $\mathbf{J}_{\mathbf{s}}$ is the distribution of source current, and the term $\bar{\sigma} \mathbf{E}$ is the induced current in the conductive earth, $\bar{\sigma}$ is the conductivity tensor which is defined as follows:

$\bar{\sigma}=\left(\begin{array}{ccc}\sigma_{x} & 0 & 0 \\ 0 & \sigma_{y} & 0 \\ 0 & 0 & \sigma_{z}\end{array}\right)$

In (3), $\sigma_{x}, \sigma_{y}, \sigma_{z}$ are principle conductivities. Actually, our formulation works for a general anisotropy case where the tensor has six independent components. For simplicity, we consider that our coordinate axes coincide with the principal axes of the conductivity tensor. In marine environment, we consider a transverse anisotropy that

$\sigma_{x}=\sigma_{y} \neq \sigma_{z}$.

In a case of a total field formulation of numerical modeling using the finite element method, the grid needs to be refined in order to capture the rapid change of the primary current. To overcome this difficulty, anomalous field formulation is desirable. In the anomalous field formulation of diffusive EM field problem, the total field is decomposed into background and anomalous fields (Zhdanov, 2009)

$\mathbf{E}=\mathbf{E}_{\mathbf{b}}+\mathbf{E}_{\mathbf{a}}$,

$\overline{\boldsymbol{\sigma}}=\bar{\sigma}_{b}+\Delta \overline{\boldsymbol{\sigma}}$.

Based on this decomposition, one can derive the following equation for the anomalous electric field:

$\nabla \times \nabla \times \mathbf{E}_{\mathbf{a}}-i \omega \mu \bar{\sigma} \mathbf{E}_{\mathbf{a}}=i \omega \mu \Delta \bar{\sigma} \mathbf{E}_{\mathbf{b}}$.

From (7), we can see that the source term for this equation is the primary electric field, which is much smoother than the source current. The normal electric field can be computed analytically for a full-space and half-space background conductivity. For a general layered earth model, the normal field can be computed semi-analytically by using a digital filter to calculate Hankel transforms.

The differential equation for anomalous electric field can be solved by using integral equation, finite difference or finite element method. Once the anomalous electric field is solved numerically, the anomalous magnetic field can be obtained by using Faraday's law (Silva et al., 2012)

$\mathbf{H}_{a}=(i \omega \mu)^{-1} \nabla \times \mathbf{E}_{\mathbf{a}}$

\section{Edge-based finite element analysis}

The edge-based finite element method uses vector basis functions defined on the edges of the corresponding elements. Similar to the conventional node-based finite element method, the modeling domain can be discretized using rectangular, tetrahedron, hexahedron or other complex elements (Jin, 2002).

For simplicity, we will discuss the rectangular grid first. Fig. 1 is an illustration of the bricks grid that we used with node and edge 

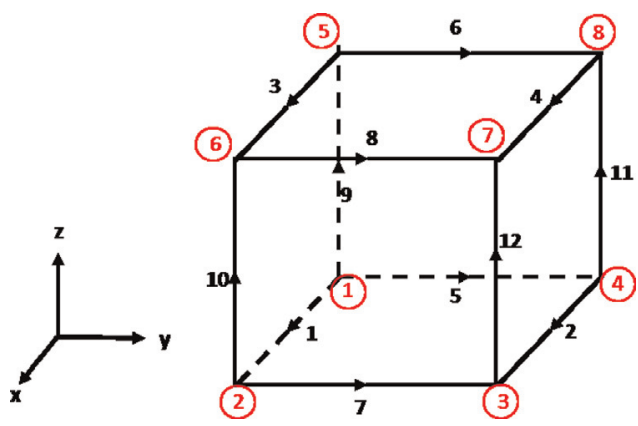

Fig. 1. An illustration of rectangular brick element. The number with circle indicates the node index and the number without circle is the index of edges.

indexing. Following the work by Jin (2002), we denote the edge length in $x, y, z$ directions as $l_{x}^{e}, l_{y}^{e}, l_{z}^{e}$ and the center of the element as $\left(x_{c}^{e}, y_{c}^{e}, z_{c}^{e}\right)$. The tangential component of the electric field is assigned to the center of each edge, the $x, y, z$ components of the electric field inside the rectangular prism can be expressed as follows:

$E_{x}^{e}=\sum_{i=1}^{4} N_{x i}^{e} E_{x i}^{e}, \quad E_{y}^{e}=\sum_{i=1}^{4} N_{y i}^{e} E_{y i}^{e}, \quad E_{z}^{e}=\sum_{i=1}^{4} N_{z i}^{e} E_{z i}^{e}$,

where the edge basis functions are defined by the following expressions (Jin, 2002):

$N_{x 1}^{e}=\frac{1}{l_{y}^{e} l_{z}^{e}}\left(y_{c}^{e}+\frac{l_{y}^{e}}{2}-y\right)\left(z_{c}^{e}+\frac{l_{z}^{e}}{2}-z\right)$,

$N_{x 2}^{e}=\frac{1}{l_{y}^{e} l_{z}^{e}}\left(y-y_{c}^{e}+\frac{l_{y}^{e}}{2}\right)\left(z_{c}^{e}+\frac{l_{z}^{e}}{2}-z\right)$,

$N_{x 3}^{e}=\frac{1}{l_{y}^{e} l_{z}^{e}}\left(y_{c}^{e}+\frac{l_{y}^{e}}{2}-y\right)\left(z-z_{c}^{e}+\frac{l_{z}^{e}}{2}\right)$,

$N_{x 4}^{e}=\frac{1}{l_{y}^{e} l_{z}^{e}}\left(y-y_{c}^{e}+\frac{l_{y}^{e}}{2}\right)\left(z-z_{c}^{e}+\frac{l_{z}^{e}}{2}\right)$

$N_{y 1}^{e}=\frac{1}{l_{z}^{e} l_{x}^{e}}\left(z_{c}^{e}+\frac{l_{z}^{e}}{2}-z\right)\left(x_{c}^{e}+\frac{l_{x}^{e}}{2}-x\right)$,

$N_{y 2}^{e}=\frac{1}{l_{z}^{e} l_{x}^{e}}\left(z-z_{c}^{e}+\frac{l_{z}^{e}}{2}\right)\left(x_{c}^{e}+\frac{l_{x}^{e}}{2}-x\right)$,

$N_{y 3}^{e}=\frac{1}{l_{z}^{e} l_{x}^{e}}\left(z_{c}^{e}+\frac{l_{z}^{e}}{2}-z\right)\left(x-x_{c}^{e}+\frac{l_{x}^{e}}{2}\right)$

$N_{y 4}^{e}=\frac{1}{l_{z}^{e} l_{x}^{e}}\left(z-z_{c}^{e}+\frac{l_{z}^{e}}{2}\right)\left(x-x_{c}^{e}+\frac{l_{x}^{e}}{2}\right)$,

$N_{z 1}^{e}=\frac{1}{l_{x}^{e} l_{y}^{e}}\left(x_{c}^{e}+\frac{l_{x}^{e}}{2}-x\right)\left(y_{c}^{e}+\frac{l_{y}^{e}}{2}-y\right)$

$$
\begin{aligned}
& N_{z 2}^{e}=\frac{1}{l_{x}^{e} l_{y}^{e}}\left(x-x_{c}^{e}+\frac{l_{x}^{e}}{2}\right)\left(y_{c}^{e}+\frac{l_{y}^{e}}{2}-y\right), \\
& N_{z 3}^{e}=\frac{1}{l_{x}^{e} l_{y}^{e}}\left(x_{c}^{e}+\frac{l_{x}^{e}}{2}-x\right)\left(z-y_{c}^{e}+\frac{l_{y}^{e}}{2}\right), \\
& N_{z 4}^{e}=\frac{1}{l_{x}^{e} l_{y}^{e}}\left(x-x_{c}^{e}+\frac{l_{x}^{e}}{2}\right)\left(y-y_{c}^{e}+\frac{l_{y}^{e}}{2}\right) .
\end{aligned}
$$

Eq. (9) can be written in a more compact form as

$\mathbf{E}^{e}=\sum_{i=1}^{12} \mathbf{N}_{i}^{e} E_{i}^{e}$,

where

$\mathbf{N}_{i}^{e}=N_{x i}^{e} \hat{x}, \quad \mathbf{N}_{i+4}^{e}=N_{y i}^{e} \hat{y}, \quad \mathbf{N}_{i+8}^{e}=N_{z i}^{e} \hat{z}$

for $i=1,2,3,4$.

It is easy to verify that the vector edge basis functions are divergence free but not curl free

$\nabla \cdot \mathbf{N}_{i}^{e}=0, \quad \nabla \times \mathbf{N}_{i}^{e} \neq \mathbf{0}$.

The vector basis functions are also continuous at the element boundaries. As a result, the divergence free condition of the electric field in the source free region and the continuity conditions are imposed directly in the edge-based finite element formulation.

By substituting (9) and (22) into (7), and using Galerkin's method, one can find the weak form of the original differential equation as follows:

$R_{i}=\int_{\Omega} \mathbf{N}_{i} \cdot\left[\nabla \times \nabla \times \mathbf{E}_{s}-i \omega \mu \bar{\sigma} \mathbf{E}_{s}-i \omega \mu \Delta \bar{\sigma} \mathbf{E}_{p}\right] d v$,

where $\omega$ is the modeling domain.

After applying the first vector Green's theorem, we can find the discretized form of (25) for each element

$R_{i}^{e}=\sum_{e=1}^{N_{e}}\left[K^{e} E_{s}^{e}-i \omega \mu M^{e} \bar{\sigma}_{e} E_{s}^{e}-i \omega \mu M^{e} \Delta \bar{\sigma}_{e} E_{p}^{e}\right]$,

where $K^{e}$ and $M^{e}$ are the local stiffness matrices defined as follows:

$K_{i j}^{e}=\int_{\Omega e}\left(\nabla \times \mathbf{N}_{i}^{e}\right) \cdot\left(\nabla \times \mathbf{N}_{j}^{e}\right) d v$,

$M_{i j}^{e}=\int_{\Omega_{e}} \mathbf{N}_{i}^{e} \cdot \mathbf{N}_{j}^{e} d v$,

and $\Omega_{e}$ indicates the domain for one element. The integrals in (27) and (28) can be calculated analytically for the rectangular elements (Jin, 2002).

After assembling the local element matrices in (26) into a global system, one can obtain a sparse linear system of equations as follows:

Ae $=\mathbf{b}$

In order to get a unique solution for this equation, proper boundary conditions need to be added. Following the work of Jin (2002) and Silva et al. (2012), we consider the homogeneous Dirichlet boundary conditions in the edge element formulation

$\mathbf{e}_{\partial \Omega}=\mathbf{0}$

which holds approximately for the anomalous electric field at a distance from the domain with the anomalous conductivity. 
For the numerical modeling, the distance, where conditions (30) hold, can be determined based on the skin depth of the field. One can refer to the work of Silva et al. (2012) and Puzyrev et al. (2013) for more details.

We use the quasi-minimum residual method with the Jacobian preconditioner to solve the linear system of (29). In order to capture the rapid change of electromagnetic field close to the source region and target area and to minimize the computational cost, we use a non-uniform rectangular grid.

As a matter of fact, there exist many other choices of iterative solvers for the large linear system of equations. The commonly used iterative solvers include GMRES and BiCGSTAB methods besides QMR. GMRES is an Arnoldi-based method which only requires one matrix-vector multiplication for the every iteration. However, this method requires large memory, because it needs all the previously generated Arnoldi vectors to be saved (Saad, 2003; Puzyrev et al., 2013). BiCGStab (Van der Vorst, 1992) and QMR (Freund and Nachtigal, 1991) are both Lanczos-based approaches. Comparing with GMRES, these two methods require two matrixvector multiplications in the every iteration, but the memory requirements for these two methods are much less comparing to the GMRES method (Puzyrev et al., 2013). In our application, the stiffness matrix is complex symmetric, and in this case the QMR method requires just one matrix-vector multiplication per iteration (Puzyrev et al., 2013; Weiss and Newman, 2002). Therefore, the QMR method is suitable for our application in terms of both computation time and memory requirements.

The convergence behavior of Krylov subspace based iterative solvers strongly depends on the conditioner number of the matrix. The computation time for solving the linear system of equations can be reduced by applying preconditioner to improve the conditioner number of the matrix (Van der Vorst, 2003). There are also many choices of preconditioners. Among these preconditioners, the Jacobian preconditioner is the simplest one which does not require extra computation (Axelsson, 1994). This type of preconditioners is demonstrated to be effective for a general case and should be used when there are no other available preconditioners (Axelsson, 1994). More advanced preconditioners based on the approximated inverse of the stiffness matrix can be used to speed up the solvers. In this paper, we have adopted the Jacobian preconditioner for simplicity and because it provided an adequate result for demonstration of our algorithm. In future, we will consider a more complex choice of the preconditioner.

We should note that the rectangular elements are not quite suitable for bathymetry modeling. It is more appropriate to use the hexahedral elements or transformed rectangular prism elements in order to simulate the bathymetry. In order to compute the stiffness matrix for the hexahedral element, we transform this element into a cubic element centered at the origin. Fig. 2a shows an arbitrary hexahedral element in the $x y z$-coordinate system, while Fig. 2b shows the transformed cubic element in the $\xi \eta \zeta$ coordinate system.

The transformation can be described by the following formulas (Jin, 2002):

$x=\sum_{i=1}^{8} N_{i}^{e}(\xi, \eta, \zeta) x_{i}^{e}$,

$y=\sum_{i=1}^{8} N_{i}^{e}(\xi, \eta, \zeta) y_{i}^{e}$,

$z=\sum_{i=1}^{8} N_{i}^{e}(\xi, \eta, \zeta) z_{i}^{e}$
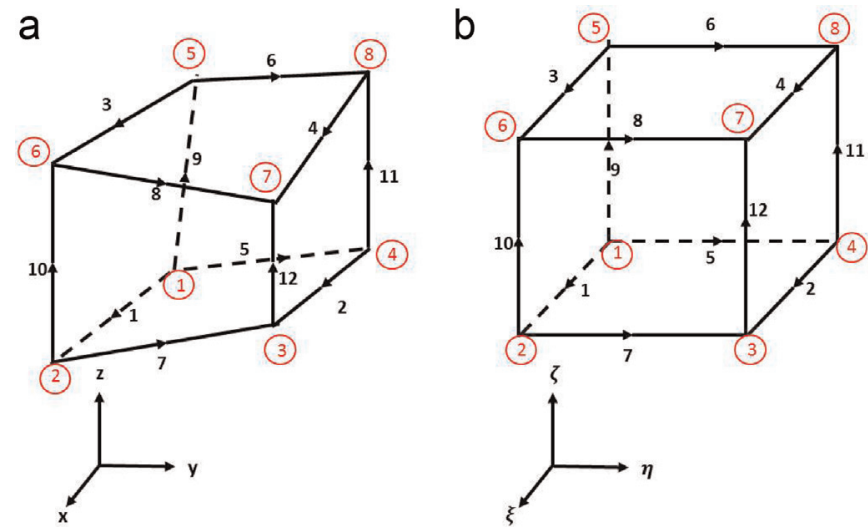

Fig. 2. (a) Hexahedral element in the $x y z$-coordinate system. (b) The same element transformed into a cubic element in the $\xi \eta \zeta$-coordinate system.

where the scalar node-based shape function, $N_{i}^{e}$, is defined as follows:

$N_{i}^{e}(\xi, \eta, \zeta)=\frac{1}{8}\left(1+\xi_{i} \xi\right)\left(1+\eta_{i} \eta\right)\left(1+\zeta_{i} \zeta\right)$

and $i$ is a local node index of the element.

The vector basis functions for the edge-based elements can be defined accordingly as follows:

$\mathbf{N}_{i}^{e}=\frac{l_{i}^{e}}{8}\left(1+\eta_{i} \eta\right)\left(1+\zeta_{i} \zeta\right) \nabla \xi, \quad 1 \leq i \leq 4$,

$\mathbf{N}_{i}^{e}=\frac{l_{i}^{e}}{8}\left(1+\xi_{i} \xi\right)\left(1+\zeta_{i} \zeta\right) \nabla \eta, \quad 5 \leq i \leq 8$,

$\mathbf{N}_{i}^{e}=\frac{l_{i}^{e}}{8}\left(1+\xi_{i} \xi\right)\left(1+\eta_{i} \eta\right) \nabla \zeta, \quad 9 \leq i \leq 12$,

where $l_{i}^{e}$ is the length of the $i$ th edge of the element.

It was shown above that for vector finite element analysis one needs to evaluate two volume integrals (27) and (28). For a hexahedral element, these two volume integrals can be transformed to the integral in the $\xi \eta \zeta$-coordinate system by using the Jacobian transform

$K_{i j}^{e}=\int_{-1}^{1} \int_{-1}^{1} \int_{-1}^{1}\left(\nabla \times \mathbf{N}_{i}^{e}\right) \cdot\left(\nabla \times \mathbf{N}_{j}^{e}\right)|J(\xi, \eta, \zeta)| d \xi d \eta d \zeta$,

$M_{i j}^{e}=\int_{-1}^{1} \int_{-1}^{1} \int_{-1}^{1} \mathbf{N}_{i}^{e} \cdot \mathbf{N}_{j}^{e}|J(\xi, \eta, \zeta)| d \xi d \eta d \zeta$,

where $J(\xi, \eta, \zeta)$ is the Jacobian matrix and $J(\xi, \eta, \zeta) \mid$ is its determinant

$J(\xi, \eta, \zeta)=\left[\begin{array}{lll}\frac{\partial x}{\partial \xi} & \frac{\partial y}{\partial \xi} & \frac{\partial z}{\partial \xi} \\ \frac{\partial x}{\partial \eta} & \frac{\partial y}{\partial \eta} & \frac{\partial z}{\partial \eta} \\ \frac{\partial x}{\partial \zeta} & \frac{\partial y}{\partial \zeta} & \frac{\partial z}{\partial \zeta}\end{array}\right]$.

The components of Jacobian matrix can be found easily by taking derivatives of (31) (through 33 ) with respect to $\xi, \eta$ and $\zeta$ coordinates

$J(\xi, \eta, \zeta)=\frac{1}{8} \sum_{k=1}^{8}\left[\begin{array}{l}\xi_{k}\left(1+\eta_{k} \eta\right)\left(1+\zeta_{k} \zeta\right)\left[\begin{array}{lll}x_{k}^{e}, & y_{k}^{e}, & z_{k}^{e}\end{array}\right] \\ \eta_{k}\left(1+\xi_{k} \xi\right)\left(1+\zeta_{k} \zeta\right)\left[\begin{array}{lll}x_{k}^{e}, & y_{k}^{e}, & z_{k}^{e}\end{array}\right] \\ \xi_{k}\left(1+\xi_{k} \xi\right)\left(1+\eta_{k} \eta\right)\left[\begin{array}{lll}x_{k}^{e}, & y_{k}^{e}, & z_{k}^{e}\end{array}\right]\end{array}\right]$. 
We can apply curl operator to (35). After doing some algebra, we can find the expression for the curl of the vector finite basis functions

$\nabla \times \mathbf{N}_{i}^{e}=\frac{l_{i}^{e}}{8}\left[\eta_{i} \nabla \eta+\zeta_{i} \nabla \zeta+\eta_{i} \zeta_{i} \eta \nabla \zeta+\eta_{i} \zeta_{i} \zeta \nabla \eta\right] \times \nabla \xi$,

$1 \leq i \leq 4$,

$\nabla \times \mathbf{N}_{i}^{e}=\frac{l_{i}^{e}}{8}\left[\xi_{i} \nabla \xi+\zeta_{i} \nabla \zeta+\xi_{i} \zeta_{i} \xi \nabla \zeta+\xi_{i} \zeta_{i} \zeta \nabla \xi\right] \times \nabla \eta$,

$5 \leq i \leq 8$,

$\nabla \times \mathbf{N}_{i}^{e}=\frac{l_{i}^{e}}{8}\left[\xi_{i} \nabla \xi+\eta_{i} \nabla \eta+\xi_{i} \eta_{i} \xi \nabla \eta+\xi_{i} \eta_{i} \eta \nabla \xi\right] \times \nabla \zeta$,

$9 \leq i \leq 12$.

Once the vector basis functions and their curl are obtained, the stiffness matrix in (36) and (37) can be evaluated numerically by using the three-dimensional Gaussian integral (Jin, 2002)

$\int_{-1}^{1} \int_{-1}^{1} \int_{-1}^{1} f(\xi, \eta, \zeta) d \xi d \eta d \zeta$

$=\sum_{i=1}^{n_{X}} \sum_{j=1}^{n_{2}} \sum_{k=1}^{n_{3}} W_{i} W_{j} W_{k} f\left(\xi_{i}, \eta_{j}, \zeta_{k}\right)$,

where $\xi_{i}, \eta_{j}$ and $\zeta_{k}$ are Gaussian integral points; $n_{1}, n_{2}$ and $n_{3}$ are the number of Gaussian integral points along $\xi, \eta$ and $\zeta$ axes; and $W_{i}, W_{j}$ and $W_{k}$ are weighting factors. In our application, the polynomial order of the integrand is less than 3 , therefore, it is sufficient to choose $n_{1}=n_{2}=n_{3}=3$ for higher accuracy. As a result, 27 Gaussian points are selected in each element to numerically compute the local stiffness matrix.

\section{Comparison with semi-analytical solution for a horizontally layered geoelectrical model}

In this section, we validate our algorithm by considering an isotropic horizontally layered geoelectrical model as shown in Fig. 3. The background is a seawater-sediment model with airearth interface at $z=0$ and the depth of seawater is $1000 \mathrm{~m}$. The conductivities of air, seawater and sediments are $10^{-6} \mathrm{Sm}^{-1}$, 3.3 $\mathrm{Sm}^{-1}$ and $1 \mathrm{Sm}^{-1}$, respectively. An infinite horizontal resistive layer with a conductivity of $0.01 \mathrm{Sm}^{-1}$ is embedded in the sediments from the depth of $1400 \mathrm{~m}$ to $1500 \mathrm{~m}$. The electromagnetic

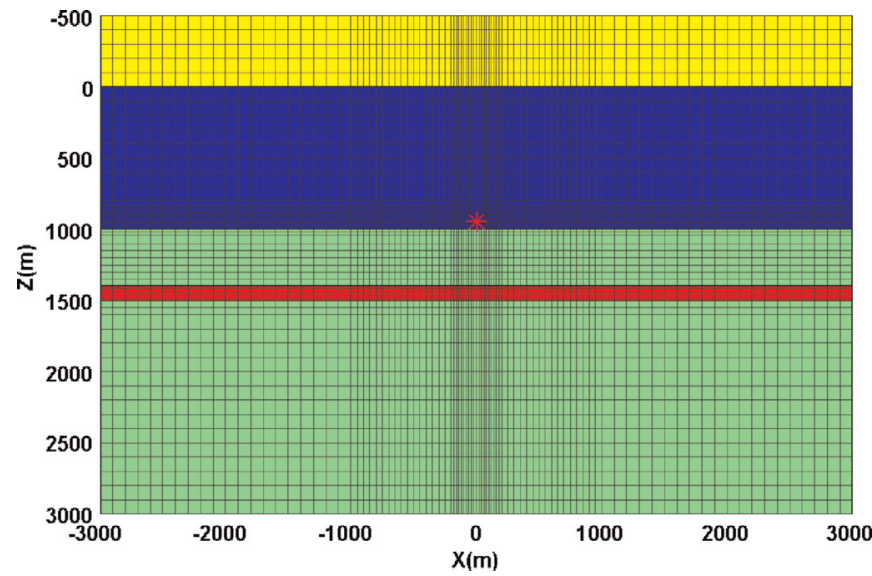

Fig. 3. Rectangular mesh for a horizontally layered geoelectrical model. The red resistive layer is embedded in sediments below seawater. The red star in this figure indicates the excitation dipole source. (For interpretation of the references to color in this figure caption, the reader is referred to the web version of this paper.)
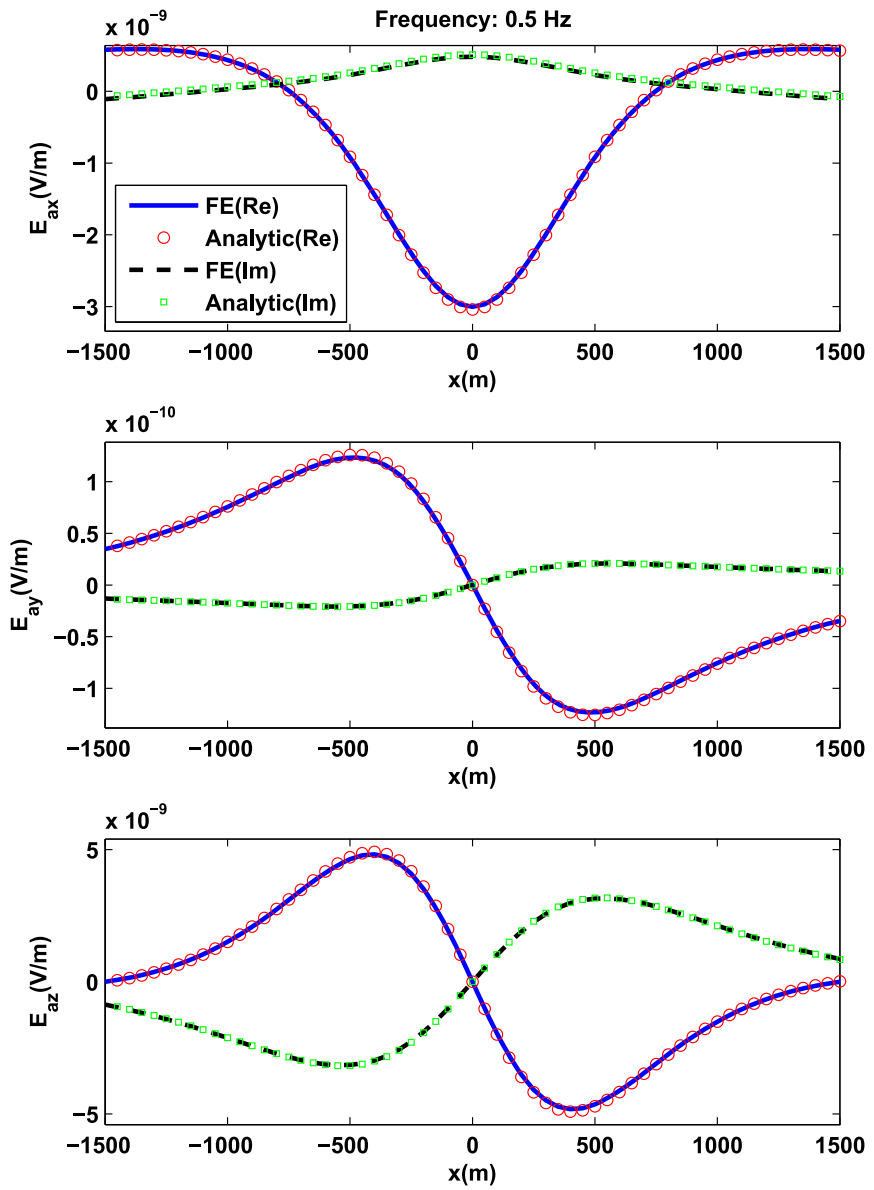

Fig. 4. A comparison between finite element result and the $1 \mathrm{D}$ semi-analytical solution for the secondary electric field with a frequency of $0.5 \mathrm{~Hz}$ on the seafloor. The upper panel shows a comparison for the $x$ component of secondary electric field at $y=0$; the middle panel shows a comparison for the $y$ component of secondary electric field at $y=-50 \mathrm{~m}$; the lower panel shows a comparison for the $z$ component of secondary electric field at $y=0$.

field is excited by an horizontal electric dipole oriented in the $x$ direction with the moment of $100 \mathrm{Am}$ and located in the seawater with the coordinates $(0,0,950) \mathrm{m}$ which is $50 \mathrm{~m}$ above seafloor. The frequency of the harmonic electric source is $0.5 \mathrm{~Hz}$. The computation domain is selected based on the skin depth criteria as $\Omega=\{-3 \mathrm{~km} \leq x, y \leq 3 \mathrm{~km} ;-0.5 \mathrm{~km} \leq z \leq 3 \mathrm{~km}\}$. We use a nonuniform rectangular grid to discretize this domain. The grid is refined nearby the source, target layer and the surface of observation (see Fig. 3).

For such an isotropic model, the anomalous field caused by the target layer can be computed semi-analytically using the Hankel transform (Ward and Hohmann, 1988; Anderson, 1989; Zhdanov and Keller, 1994; Guptasarma and Singh, 1997). Fig. 4 shows a comparison of the anomalous electric field between the finite element solution and the 1D semi-analytical solution. Fig. 5 shows a comparison of the anomalous magnetic field between the finite element solution and the 1D semi-analytical solution. One can see that the finite element results are in a good agreement with the semi-analytical solution. For this model with the specified source configuration, the $y$ component of secondary electric field, the $x$ and $z$ components of secondary magnetic field are equal to 0 at $y=0$. As such, the finite element solution of these three components are compared with the $1 \mathrm{D}$ semi-analytical solution at $y=-50 \mathrm{~m}$ where their values are not 0 . 

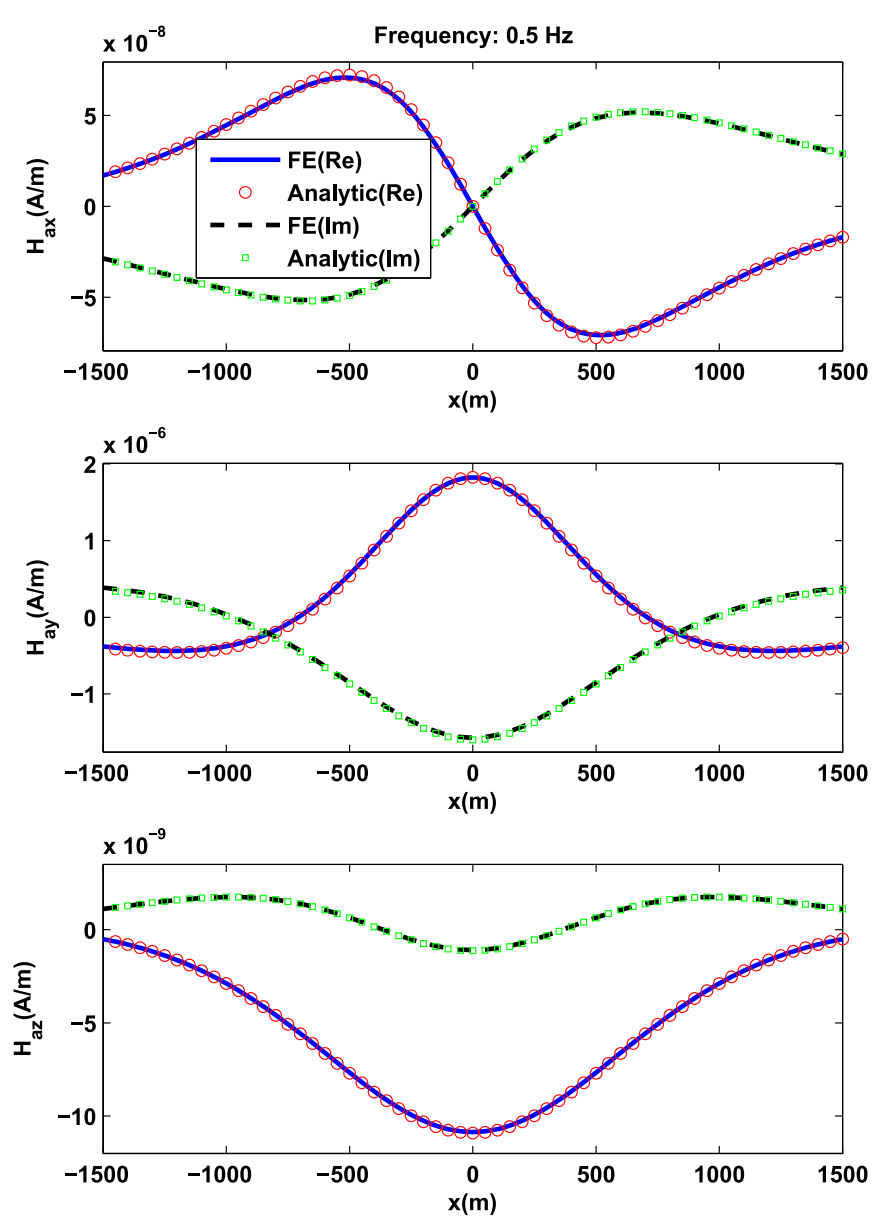

Fig. 5. A comparison between the finite element result and the 1D semi-analytical solution for the secondary magnetic field with a frequency of $0.5 \mathrm{~Hz}$ on the seafloor. The upper panel shows a comparison for the $x$ component of secondary magnetic field at $y=-50 \mathrm{~m}$; the middle panel shows a comparison for the $y$ component of secondary magnetic field at $y=0$; the lower panel shows a comparison for the $z$ component of secondary magnetic field at $y=-50 \mathrm{~m}$.

\section{Model of an off-shore hydrocarbon reservoir}

In this section, we consider a 3D model of a hydrocarbon (HC) reservoir in a marine environment. We consider a three-layered background model where the first layer is air with a conductivity of $10^{-6} \mathrm{Sm}^{-1}$, the second layer is seawater with a conductivity of 3.3 $\mathrm{Sm}^{-1}$ and the bottom layer is sediment with a horizontal conductivity of $1 \mathrm{Sm}^{-1}$. The horizontal conductivity of the reservoir is set to be $0.05 \mathrm{Sm}^{-1}$. The seawater depth is $1000 \mathrm{~m}$ and it is separated from the sediment by a horizontal flat plane. The anomalous domain for the resistive $\mathrm{HC}$ reservoir is defined as follows: $\Omega_{a}=\{-1000 \mathrm{~m} \leq x, y \leq 1000 \mathrm{~m} ; 2000 \mathrm{~m} \leq z \leq 2100 \mathrm{~m}\}$.

The transmitter is an $x$-oriented electric dipole source located $100 \mathrm{~m}$ above the seafloor at a point with the coordinates $(-3000,0,900) \mathrm{m}$. The frequency of excitation current is $1 \mathrm{~Hz}$, which is a typical frequency for marine CSEM. The EM receivers are located on the seafloor. The computation domain for finite element analysis is selected as $\Omega=\{-5 \mathrm{~km} \leq x \leq 5 \mathrm{~km}$; $-3.6 \mathrm{~km} \leq y \leq 3.6 \mathrm{~km} ;-1 \mathrm{~km} \leq z \leq 3.5 \mathrm{~km}\}$. Fig. 6 shows a $3 \mathrm{D}$ view of the horizontal conductivity for this model of the off-shore HC reservoir.

The computation domain is discretized by a non-uniform rectangular grid. Figs. 7 and 8 show the grid in the $X-Z$ plane at $y=0$ and the grid in a plane view, respectively. From these figures, one can see that the mesh is refined in the areas of the dipole

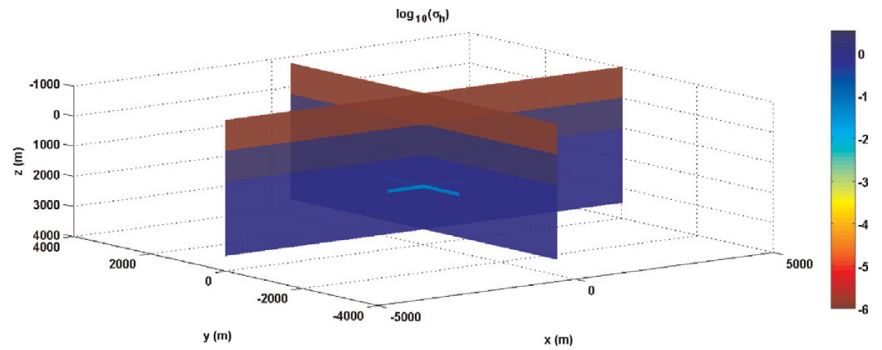

Fig. 6. 3D view of a model of the off-shore HC reservoir. The resistivity is shown in logarithmic scale.

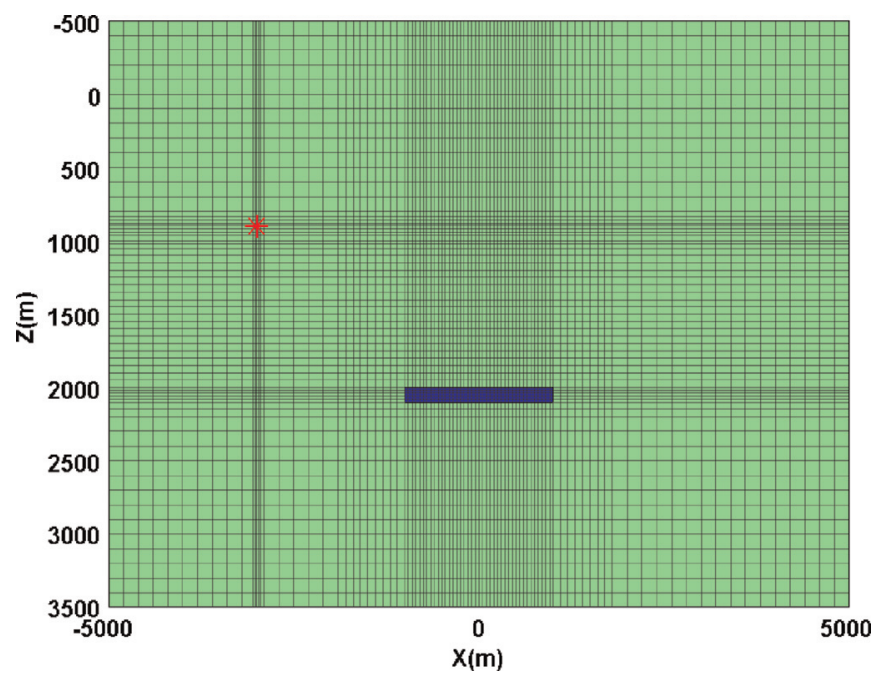

Fig. 7. Vertical section at $y=0$ of the grid used for the model of the off-shore HC reservoir. The blue area indicates the reservoir and the red star represents the location of a dipole source. (For interpretation of the references to color in this figure caption, the reader is referred to the web version of this paper.)

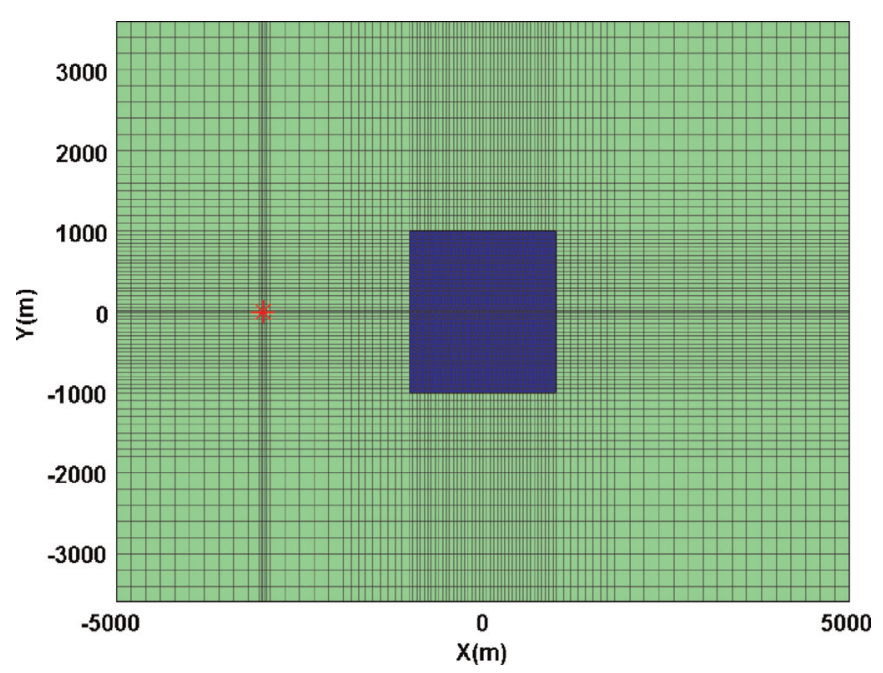

Fig. 8. Plane view of the grid used for the model of the off-shore HC reservoir. The blue area indicates the reservoir and the red star represents the location of a dipole source. (For interpretation of the references to color in this figure caption, the reader is referred to the web version of this paper.)

source, reservoir domain, and the seafloor, where the data are measured by the receivers.

This mesh contains 438,216 elements, 455,994 nodes and $1,349,971$ edges. Thus, the degree of freedom associated with the 

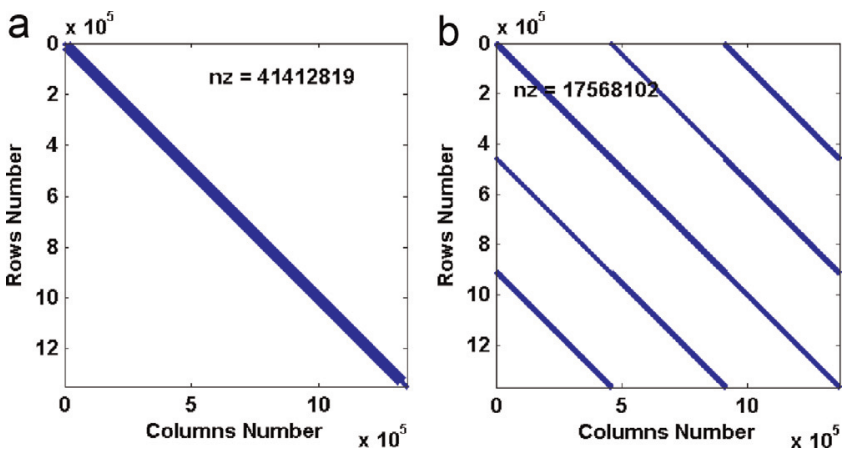

Fig. 9. Sparsity patterns of the FEM and FDM stiffness matrices for the 3D reservoir model. Panel (a) shows the sparsity pattern of edge-based finite element stiffness matrix (with 41,412,819 nozero entries), while panel (b) presents the sparsity of finite difference stiffness matrix (with 17,568,102 nozero entries) for the same model.

edges is 1349.971 . The resulting size of the sparse stiffness matrix is $1,349,971 \times 1,349,971$. The memory required to store this sparse matrix is around $1 \mathrm{~GB}$. The sparsity pattern for the finite element stiffness matrix is shown in panel (a) of Fig. 9. We also create a stiffness matrix using the finite difference method for the same model and the sparsity pattern is shown in panel of Fig. 9. From this figure, one can see that the matrix is very sparse, although the problem size is huge. The number of nonzero entries of the finite element stiffness matrix is almost 2.3 times as that of the nonzero entries of the finite difference stiffness matrix. However, the nonzero entries of the finite element stiffness matrix are more centralized which results in a narrower bandwidth of the matrix.

In this section, we will consider four sub-models with the same geometry but with different anisotropy configurations. In the first model, we assume that both the background and anomalous conductivities are isotropic. In the second model, we consider an anisotropic background conductivity and isotropic anomalous conductivity for the reservoir. In the third model, we assume that the background is isotropic while the reservoir is anisotropic. Finally, we will consider a more complex case (Model 4), where both the background and anomalous conductivities are anisotropic.

It is known that marine CSEM data have lower sensitivity to horizontal conductivity in comparison to the vertical conductivity (e.g., Brown et al., 2012). In our model studies for anisotropic reservoir, the horizontal conductivity is fixed while the vertical conductivity changes. Tompkins (2005), Li and Dai (2011) and Brown et al. (2012) show that the sensitivity of MCSEM data to reservoir anisotropy is very low while the anisotropic background conductivity can affect the anomalous fields significantly. In order to compare the anisotropy effects from background and reservoir conductivities, we set larger anisotropy ratio for reservoir conductivity than for background conductivity.

We will compare the numerical modeling results based on the edge element analysis which will be produced by the integral equation method (Zhdanov et al., 2006).

\subsection{Model 1: isotropic background and isotropic reservoir}

Model 1 has isotropic conductivity both in the layered background and within the reservoir of $1 \mathrm{Sm}^{-1}$ and $0.05 \mathrm{Sm}^{-1}$, respectively. The numerical result obtained by the edge-based finite element method was compared with the integral equation solution. Fig. 10 shows, as an example, a comparison of $E_{x}$ component computed by these two methods along the profile at $y=0$ with the frequency of $1 \mathrm{~Hz}$. We can see that the result obtained by the
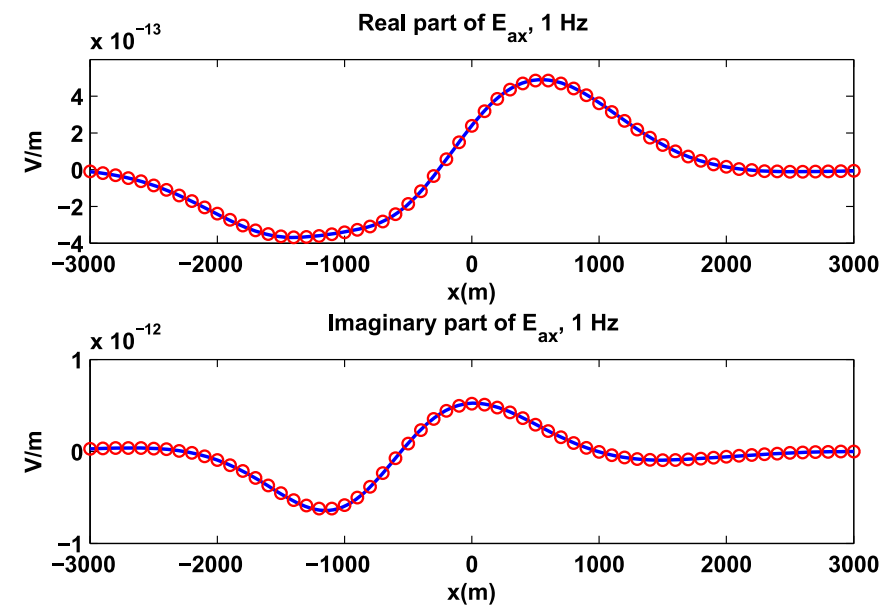

Fig. 10. Model 1. A comparison of the $x$ component of the anomalous electric field computed using the finite element (red circles) and integral equation (blue line) methods at $y=0$ for a frequency of $1 \mathrm{~Hz}$. (For interpretation of the references to color in this figure caption, the reader is referred to the web version of this paper.)

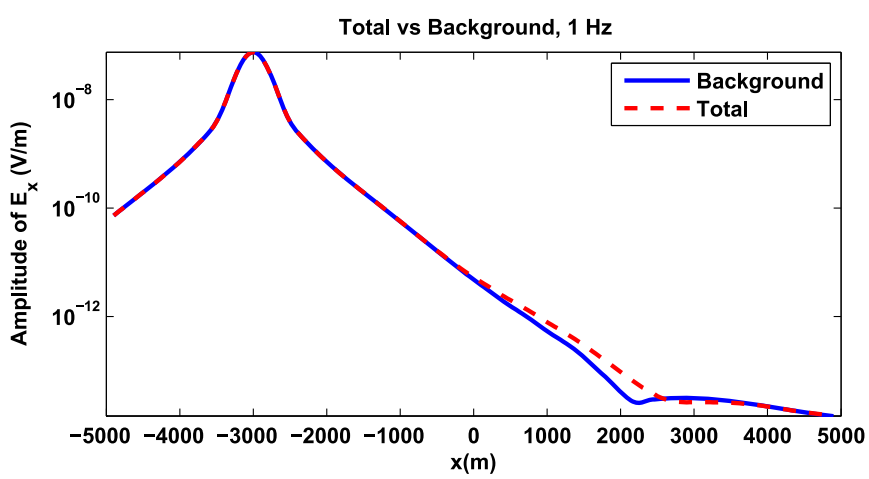

Fig. 11. Model 1. A comparison of the $x$ component of the background and total electric fields at $y=400 \mathrm{~m}$ for a frequency of $1 \mathrm{~Hz}$. The solid blue line shows the background field while the dashed red line represents the total field. (For interpretation of the references to color in this figure caption, the reader is referred to the web version of this paper.)

integral equation method is practically the same as the finite element solution. Fig. 11 shows the plots of the background and total $E_{x}$ components with the frequency of $1 \mathrm{~Hz}$ for the same model. One can clearly see an anomaly around $x=2 \mathrm{~km}$ where the offset is about $5 \mathrm{~km}$. Within the $3 \mathrm{~km}$ offset, the total field is almost the same as the background field since the anomalous field becomes much smaller than the background field for the receivers located closer to the source.

In order to further demonstrate our algorithm, we added the comparison of finite element solution and integral equation solution for another two frequencies: $0.1 \mathrm{~Hz}$ and $0.5 \mathrm{~Hz}$. Figs. 12 and 13 show the comparison of $E_{x}$ component computed by finite element and integral equation methods along the profile at $y=0$ with the frequencies of $0.1 \mathrm{~Hz}$ and $0.5 \mathrm{~Hz}$, respectively. Due to the page limits, we will only show the numerical modeling result for the frequency of $1 \mathrm{~Hz}$ in the following sections.

Fig. 14 shows the convergence plot of QMR solver for this model for the data with the frequency of $1 \mathrm{~Hz}$. We also present the convergence plots of the GMRES and BiCGSTAB solvers in this figure. We can clearly see that QMR solver is more stable comparing to BiCGSTAB solver and the convergence rate is faster than that for GMRES and BiCGSTAB solvers. We obtain similar results for other models presented in this paper. Due to the page limits, we only show a comparison of the convergences of QMR, GMRES and BiCGSTAB solvers for Model 1. 

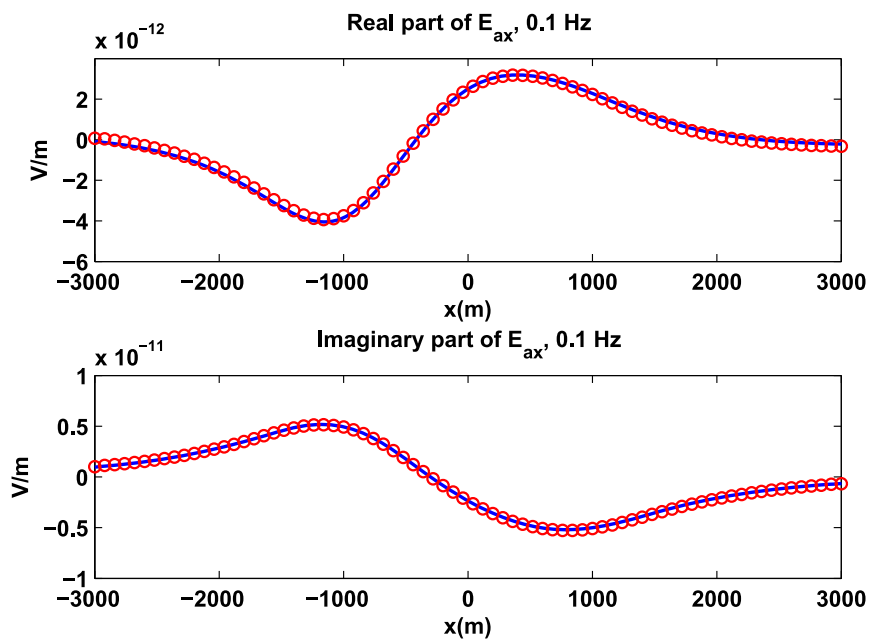

Fig. 12. Model 1. A comparison of the $x$ component of the anomalous electric field computed using the finite element (red circles) and integral equation (blue line) methods at $y=0$ for a frequency of $0.1 \mathrm{~Hz}$. (For interpretation of the references to color in this figure caption, the reader is referred to the web version of this paper.)
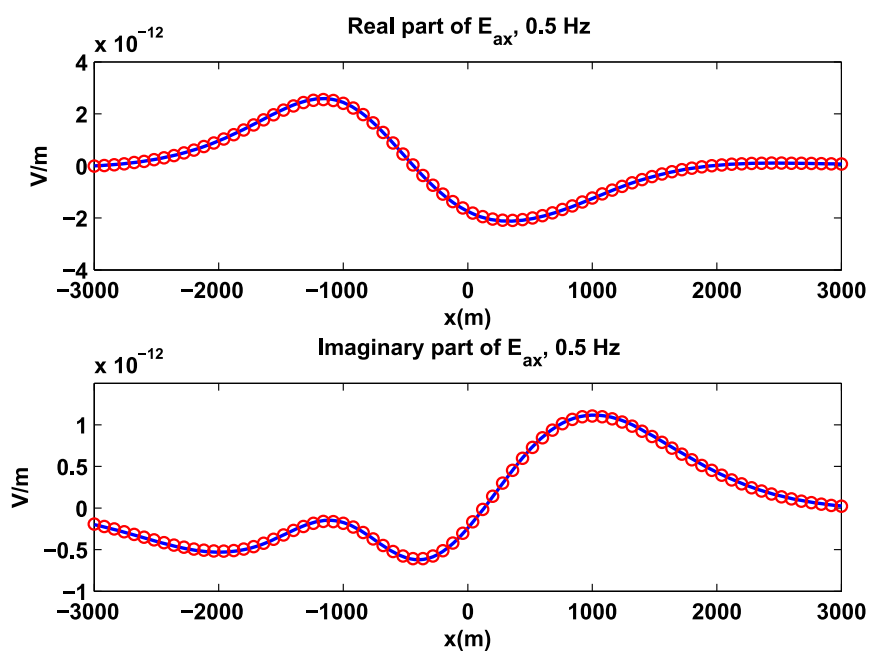

Fig. 13. Model 1. A comparison of the $x$ component of the anomalous electric field computed using the finite element (red circles) and integral equation (blue line) methods at $y=0$ for a frequency of $0.5 \mathrm{~Hz}$. (For interpretation of the references to color in this figure caption, the reader is referred to the web version of this paper.)

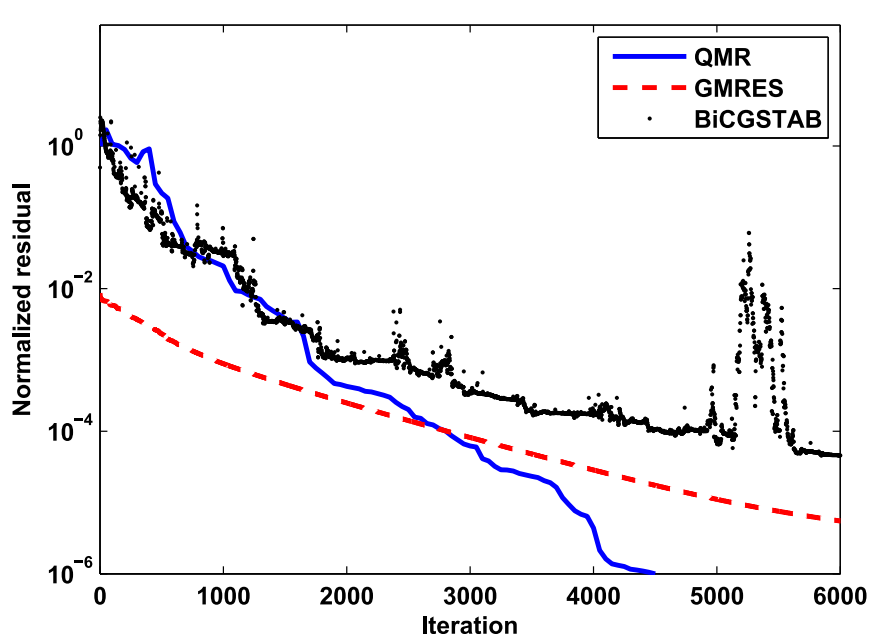

Fig. 14. Convergence plot of QMR solver for 3D reservoir Model 1 for the data with the frequency of $1 \mathrm{~Hz}$ compared to the convergence plots of the GMRES and BiCGSTAB solvers.
The total memory required to solve this model using finite element and integral equation methods was $1.5 \mathrm{~GB}$ and $215 \mathrm{MB}$, respectively. It took about $20 \mathrm{~min}$ to solve this model using the finite element method and about 3 min for the integral equation method on a PC with $2.6 \mathrm{GHz}$ CPU.

\subsection{Model 2: anisotropic background with isotropic reservoir}

In a marine environment, the conductivity of sediments shows a strong transverse anisotropy due to the process of sedimentation with the longitudinal conductivity being larger than the transverse conductivity (Ellis et al., 2010; Ramananjaona et al., 2011). Specifically, there are two major causes of such transverse conductivity: thin layering and grain alignment (Ellis et al., 2010). The macroanisotropy is mainly caused by thin layering when bulk resistivity is measured so that the electric current prefers to travel parallel to the bedding planes (Ellis et al., 2010). The micro-anisotropy at the grain scale results from the preferred mineral alignment, most often due to compaction in the process of sedimentation, for example in shale (Clavaud, 2008; Ramananjaona et al., 2011). This transverse anisotropy could have a strong effect on the primary field and the anomalous field could also be distorted significantly. The effect of anisotropy on EM field has been discussed in a number of publications (e.g., Løseth and Ursin, 2007).

In Model 2, we have selected the following horizontal and vertical conductivities of the sea-bottom sediments: $\sigma_{h}=\sigma_{x}=\sigma_{y}=1 \mathrm{Sm}^{-1}$, $\sigma_{z}=0.8 \mathrm{Sm}^{-1}$. At the same time, we assume that the reservoir conductivity is isotropic, $\sigma_{a x}=\sigma_{a y}=\sigma_{a z}=0.05 \mathrm{Sm}^{-1}$.

The conductivity of seawater and air stays unchanged compared to the previous model. The conductivity anisotropy coefficient $(\lambda)$ is defined as the square root of the ratio of the longitudinal conductivity $\left(\sigma_{h}\right)$ to the transverse conductivity $\left(\sigma_{z}\right)$ (Negi and Saraf, 1989)

$\lambda_{0}=\sqrt{\frac{\sigma_{h}}{\sigma_{z}}} \approx 1.12$.

As in the previous model, we compare the finite element result with the integral equation solution. Fig. 15 shows a comparison of the fields computed by these two methods at $y=0$ with the frequency of $1 \mathrm{~Hz}$. We can see that the result obtained by the integral equation method is practically the same as the finite element solution for this model. By comparing Fig. 10 with Fig. 15, we observed a strong distortion of the anomalous field by the anisotropy of the background conductivity.
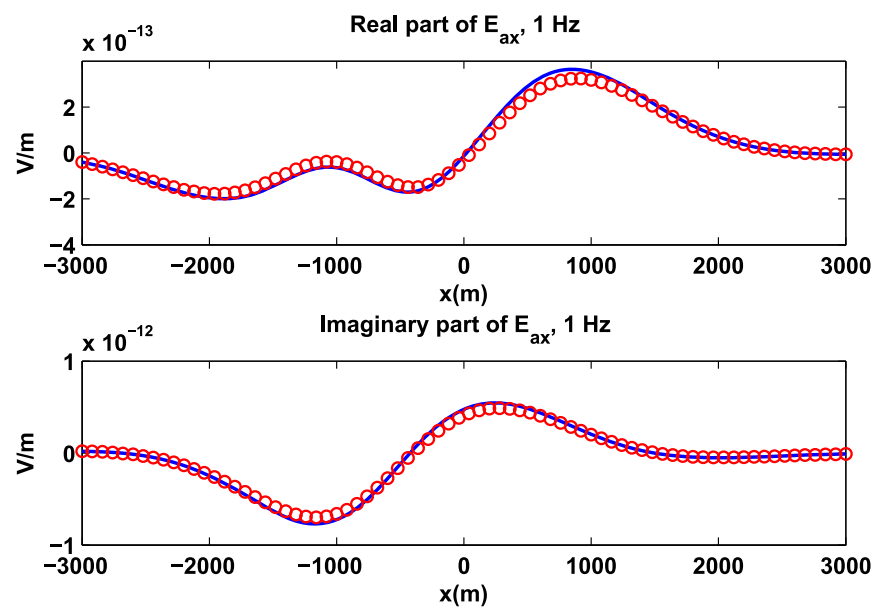

Fig. 15. Model 2. A comparison of the $x$ component of anomalous electric field computed using the finite element (red circles) and integral equation (blue line) methods at $y=0$ for a frequency of $1 \mathrm{~Hz}$. (For interpretation of the references to color in this figure caption, the reader is referred to the web version of this paper.) 


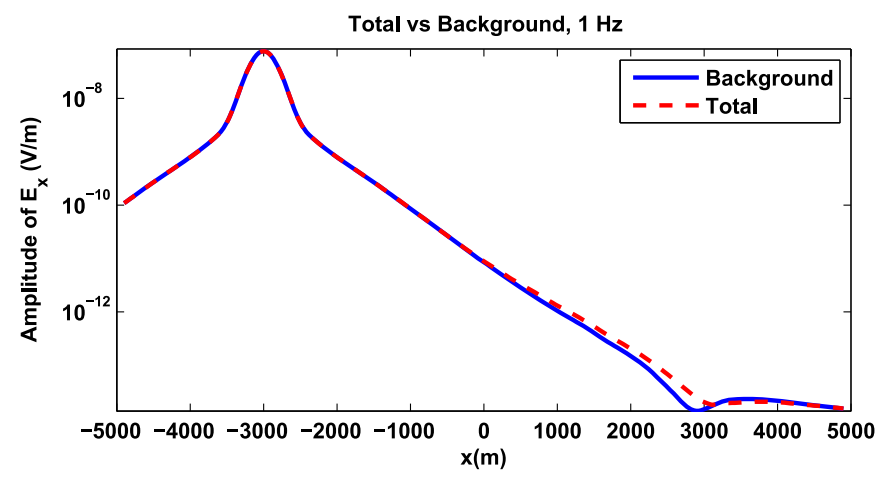

Fig. 16. Model 2. A comparison of the $x$ component of the background and total electric fields at $y=400 \mathrm{~m}$ for a frequency of $1 \mathrm{~Hz}$. The solid blue line shows the background field while the dashed red line represents the total field. (For interpretation of the references to color in this figure caption, the reader is referred to the web version of this paper.)

Fig. 16 presents $E_{x}$ component of the background and total electric fields with the frequency of $1 \mathrm{~Hz}$. A comparison between the plots of Figs. 11 and 16 shows that the anomaly related to the $\mathrm{HC}$ reservoir is shifted to the right for the anisotropic background model.

The total memory requirement for solving this problem using finite element and integral equation methods were the same as for Model 1. It took about 22 min to solve this model using the finite element method and about $3 \mathrm{~min}$ for the integral equation method on a PC with $2.6 \mathrm{GHz}$ CPU.

\subsection{Model 3: isotropic background with anisotropic reservoir}

In practical applications of the MCSEM method, not only the conductivity of the sea-bottom sediments, but also the reservoir conductivity can be anisotropic. In this model, we will examine the effect of anisotropy of the conductivity of the $\mathrm{HC}$ reservoir on the anomalous field.

We assume first that the background conductivity is isotropic: $\sigma_{x}=\sigma_{y}=\sigma_{z}=1 \mathrm{Sm}^{-1}$. The reservoir anisotropy is usually weak in comparison to the background conductivity anisotropy (Brown et al., 2012). As we can see from the previous model with anisotropic background conductivity, the anomalous EM field is distorted by the background anisotropy significantly. In order to better see the distortions of anomalous field caused by reservoir anisotropy, we set a large anisotropy coefficient for the conductivity of reservoir in Model 3. The horizontal and vertical conductivities of the reservoir are set to the following values: $\sigma_{a h}=\sigma_{a x}=\sigma_{a y}=0.05 \mathrm{Sm}^{-1}$ and $\sigma_{a z}=0.005 \mathrm{Sm}^{-1}$, respectively. The anisotropy coefficient is equal to

$\lambda_{a}=\sqrt{\frac{\sigma_{a h}}{\sigma_{a z}}} \approx 3.2$.

Fig. 17 shows a comparison of the fields computed by the finite element and integral equation methods at $y=0$ with the frequency of $1 \mathrm{~Hz}$. We can see that the result obtained by the integral equation method is practically the same as the finite element solution for the anisotropic reservoir model. Fig. 18 presents $E_{X}$ components of the background and total electric field with the frequency of $1 \mathrm{~Hz}$.

The total memory requirement for solving this problem using finite element and integral equation methods were practically the same as for Model 1. It took about $25 \mathrm{~min}$ to solve this problem using the finite element method and about $4 \mathrm{~min}$ for the integral equation method on a PC with $2.6 \mathrm{GHz}$ CPU.
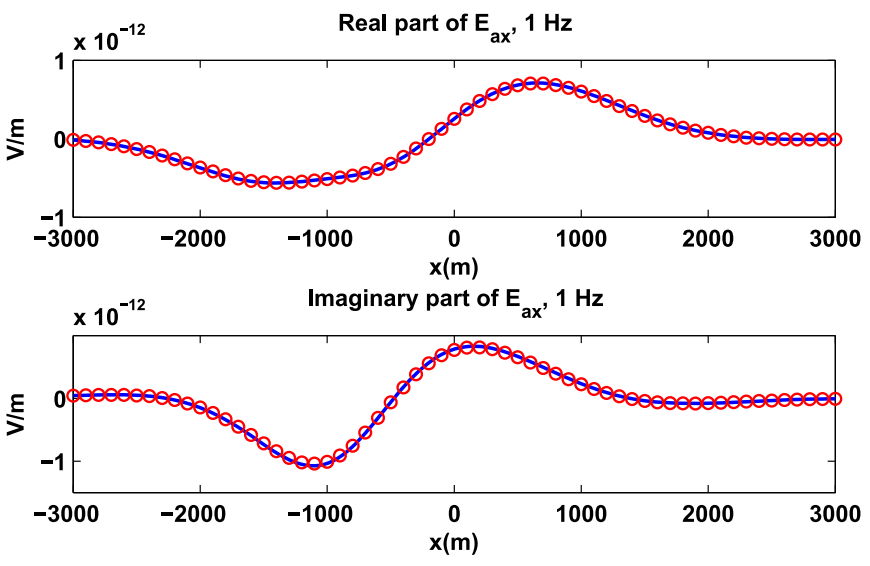

Fig. 17. Model 3. A comparison of the $x$ component of anomalous electric field computed using the finite element (red circles) and integral equation (blue line) methods at $y=0$ for a frequency of $1 \mathrm{~Hz}$. (For interpretation of the references to color in this figure caption, the reader is referred to the web version of this paper.)

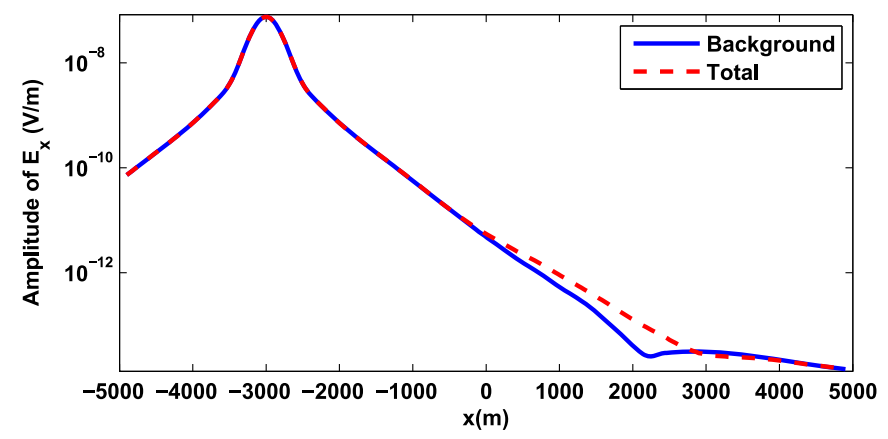

Fig. 18. Model 3. A comparison of the $x$ component of the background and tota electric fields at $y=400 \mathrm{~m}$ for a frequency of $1 \mathrm{~Hz}$. The solid blue line shows the background field while the dashed red line represents the total field. (For interpretation of the references to color in this figure caption, the reader is referred to the web version of this paper.)

\subsection{Model 4: anisotropic background with anisotropic reservoir}

Finally, we study Model 4 having transverse anisotropy of both the background and reservoir conductivities. This model is a combination of Model 2 and Model 3. We set the background conductivity equal to $\sigma_{h}=\sigma_{x}=\sigma_{y}=1 \mathrm{Sm}^{-1}, \sigma_{z}=0.8 \mathrm{Sm}^{-1}$ and the reservoir conductivity to be $\sigma_{a h}=\sigma_{a x}=\sigma_{a y}=0.05 \mathrm{Sm}^{-1}, \sigma_{a z}=0.005 \mathrm{Sm}^{-1}$. For this model
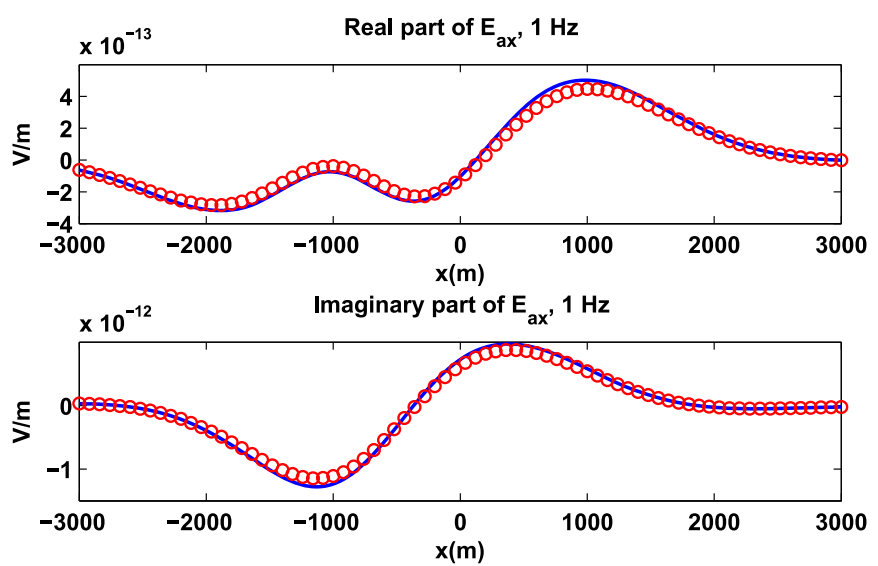

Fig. 19. Model 4. A comparison of the $x$ component of anomalous electric field computed using the finite element (red circles) and integral equation (blue line) methods at $y=0$ for a frequency of $1 \mathrm{~Hz}$. (For interpretation of the references to color in this figure caption, the reader is referred to the web version of this paper.) 


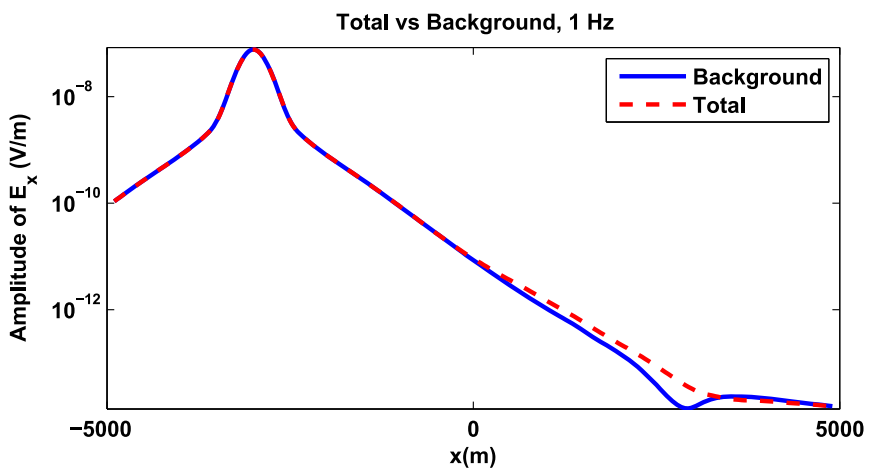

Fig. 20. Model 4. A comparison of the $x$ component of the background and total electric fields at $y=400 \mathrm{~m}$ for a frequency of $1 \mathrm{~Hz}$. The solid blue line shows the background field while the dashed red line represents the total field. (For interpretation of the references to color in this figure caption, the reader is referred to the web version of this paper.)

the anisotropy coefficients for the background and reservoir conductivities are equal to approximately 1.12 and 3.16, respectively.

Fig. 19 shows the plots of the $x$ components of electric field computed at $y=0$ with the frequency of $1 \mathrm{~Hz}$. The results produced by the integral equation method coincide well with the finite element solution. Fig. 20 presents $E_{x}$ component of the background and total field with the frequency of $1 \mathrm{~Hz}$, with the anomaly observed around a point $x=3 \mathrm{~km}$, which corresponds to the $6 \mathrm{~km}$ offset.

The total memory requirement of this problem for finite element and integral equation methods are the same as Model 1. It took about 30 min to solve this model using the finite element method and around 5 min for the integral equation method on a PC with $2.6 \mathrm{GHz}$ CPU.

Fig. 21 presents a comparison of the amplitudes of the anomalous electric field normalized by the background field for Models 1, 2, 3 and 4 at $y=0$ with the frequency of $1 \mathrm{~Hz}$. One can see that for these models that the anomalous field is distorted by anisotropy of both background and reservoir conductivities. The effect of the anisotropy of background conductivity is manifested by shifting the anomaly to larger offset, while the increase of the anisotropy coefficient of the reservoir increases the amplitude of the anomaly without shifting the anomaly significantly. Thus, our numerical modeling results confirm ones again that quantitative interpretation of the MCSEM data requires taking into account the effect of anisotropy on the observed data.

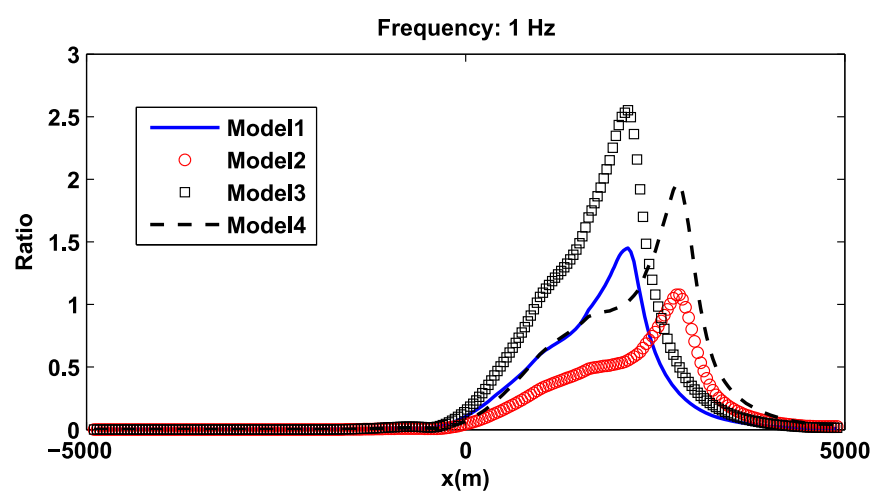

Fig. 21. A comparison of normalized anomalous field at $y=0$ for different $3 D$ models for a frequency of $1 \mathrm{~Hz}$.

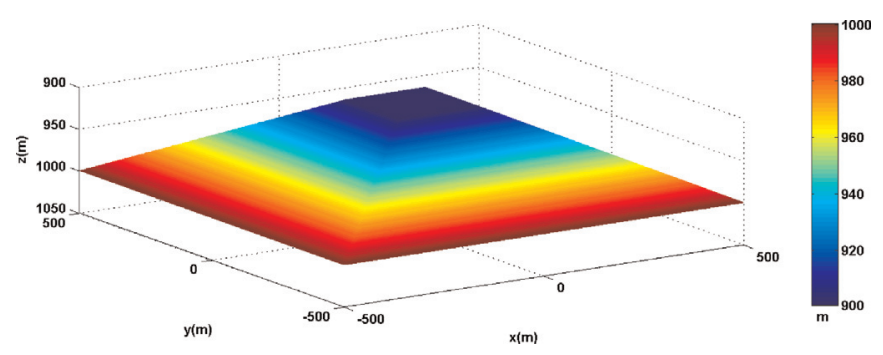

Fig. 22. A 3D view of a trapezoidal-type hill model of the seafloor bathymetry.

\section{Modeling the effect of the bathymetry on the EM data}

One of the advantages of the edge-based finite element method is its ability to model the bathymetry effect on the EM data. The non-uniform rectangular elements can be applied to simulate very simple bathymetry by using a staircase approximation in a similar way as in the framework of finite difference or integral equation approaches. In order to simulate more complex seafloor topography, one needs to use more flexible hexahedral or tetrahedral elements. There are exist well-developed software to generate unstructured tetrahedral mesh. However, there is still no automatic mesh generator available for unstructured hexahedral mesh.

As shown in Fig. 22, we consider a trapezoidal-type hill model of the seafloor bathymetry. The domain with bathymetry variations is denoted by $\Omega=\{-500 \mathrm{~m} \leq x, y \leq 500 \mathrm{~m}\}$. We assume also that, in domain $\Omega_{1}=\{-100 \mathrm{~m} \leq x, y \leq 100 \mathrm{~m}\}$, the sea floor is flat and located at a depth of $z=900 \mathrm{~m}$, which is $100 \mathrm{~m}$ above the flat seafloor model considered in the previous section. Within other area of domain $\Omega$, the seafloor depth changes from $900 \mathrm{~m}$ to $1000 \mathrm{~m}$ linearly. Fig. 22 is an illustration of the trapezoidal hill model. Outside domain $\omega$, the seafloor is flat and is located at a depth of $1 \mathrm{~km}$. In order to generate the hexahedral mesh, we have transformed the original nonuniform rectangular grid by shifting the $z$-coordinate of the nodes to accommodate bathymetry.

We first consider a bathymetry model without a reservoir. Fig. 23 shows the hexahedral grid for the bathymetry model without a reservoir in the $X-Z$ section at $y=0$. The conductivities of the air, seawater, and sediment are the same as we described in the previous section. We assume for simplicity that this model is isotropic. The anomalous field caused by the variations of bathymetry is computed using both the edge-based finite element and integral equation methods. For integral equation approach, the bathymetry is approximated using a staircase model, which is shown in Fig. 24. We use a very fine discretization of $5 \mathrm{~m}$ in the vertical direction for the staircase model. We consider an $x$-oriented electric dipole source located at the point with coordinates $(-1500,0,900) \mathrm{m}$. The frequency of excitation current is $1 \mathrm{~Hz}$.

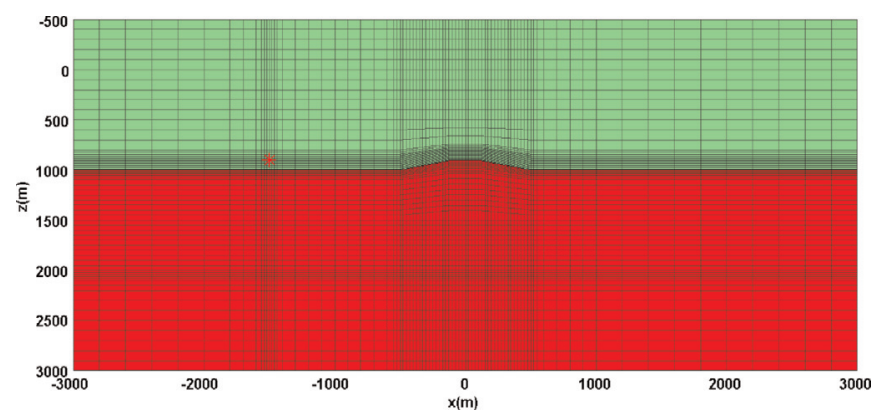

Fig. 23. $X-Z$ section of the hexahedral grid for bathymetry model without a reservoir. The red star indicates the location of the dipole source oriented in the $x$ direction. (For interpretation of the references to color in this figure caption, the reader is referred to the web version of this paper.) 


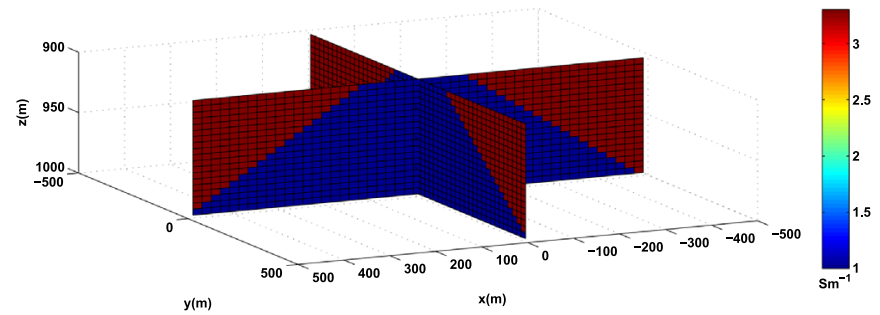

Fig. 24. A staircase approximation of the bathymetry model use in the framework of the integral equation method.
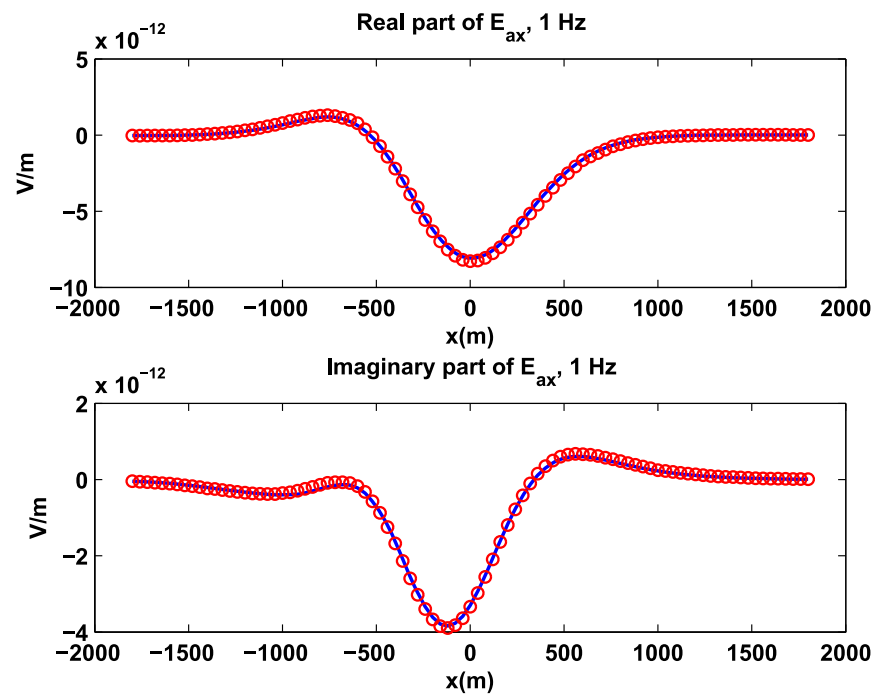

Fig. 25. Bathymetry model without $\mathrm{HC}$ reservoir. A comparison of the $x$ component of the anomalous electric field computed using the finite element (red circles) and integral equation (blue line) methods at $y=0$ and $z=500 \mathrm{~m}$ for a frequency of $1 \mathrm{~Hz}$. (For interpretation of the references to color in this figure caption, the reader is referred to the web version of this paper.)

Since the nodes of original rectangular and new hexahedral grids are the same at a depth of $z=500 \mathrm{~m}$, we first compare the fields at $z=500 \mathrm{~m}$. Fig. 25 shows a comparison of the anomalous field at $y=0$ and $z=500 \mathrm{~m}$, with the frequency of $1 \mathrm{~Hz}$, computed using edge-based finite element and integral equation methods.
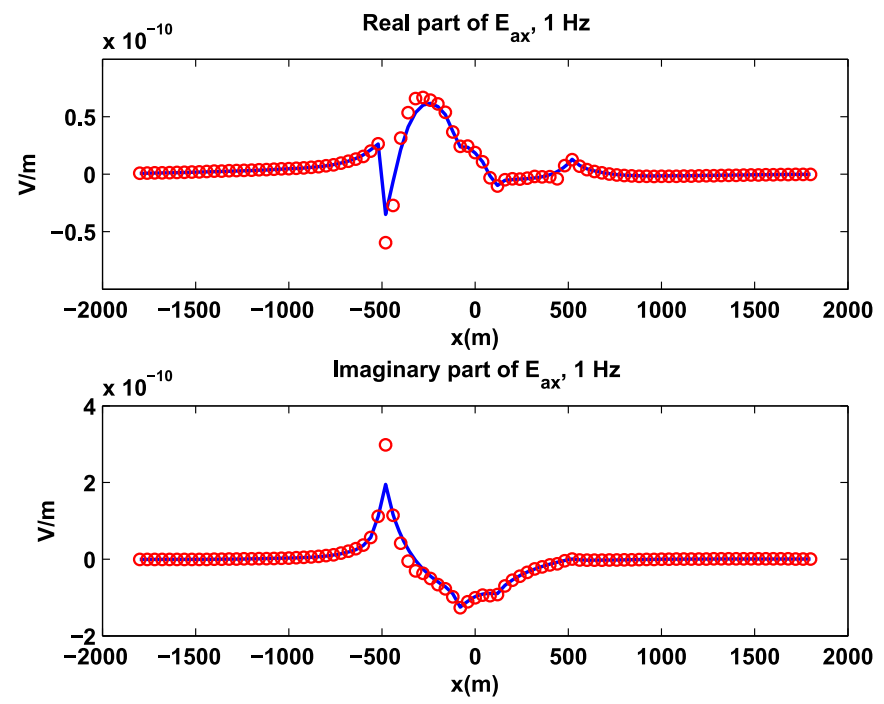

Fig. 26. Bathymetry model without $\mathrm{HC}$ reservoir. A comparison of the $x$ component of the anomalous electric field computed using the finite element (red circles) and integral equation (blue line) methods at $y=0$ along the sea floor for a frequency of $1 \mathrm{~Hz}$. (For interpretation of the references to color in this figure caption, the reader is referred to the web version of this paper.)

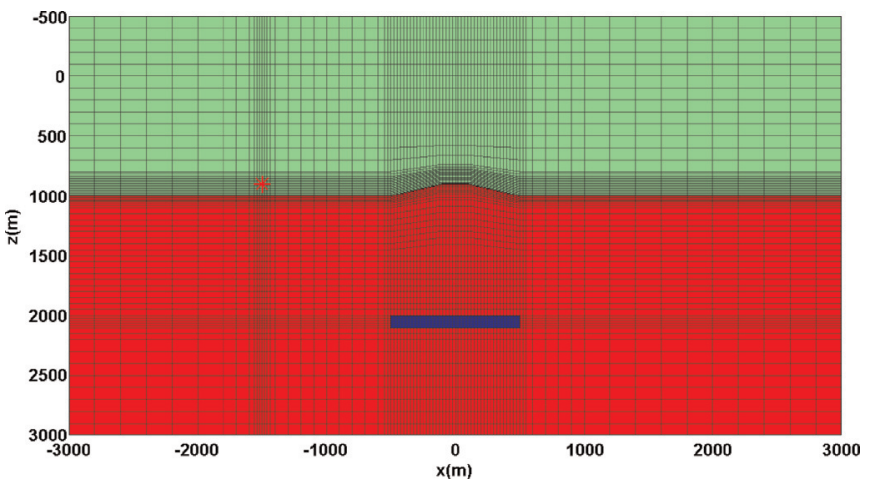

Fig. 27. $X-Z$ section of the hexahedral grid for bathymetry model with a reservoir The red star indicates the location of the dipole source oriented in the $x$ direction. (For interpretation of the references to color in this figure caption, the reader is referred to the web version of this paper.)

We can see that the results produced by these two methods are practically coincide. Thus, if the receivers are located away from the bathymetry, a staircase model is a good approximation of the bathymetry for the integral equation method.

Fig. 26 shows a comparison of the anomalous field at $y=0$ along the bathymetry, with the frequency of $1 \mathrm{~Hz}$, computed using both the edge-based finite element and integral equation methods. One can see that the results of these two methods still show a good agreement with each other. We observe some difference between the results of these two methods at the boundary of the bathymetry variation $(x=-500 \mathrm{~m})$ only. This difference can be attributed to the staircase approximation used in the integral equation method.

For this bathymetry model without a reservoir, the total memory required to solve this model using finite element and integral equation methods are $1.8 \mathrm{~GB}$ and $300 \mathrm{MB}$, respectively. It took about $25 \mathrm{~min}$ to solve this model using the finite element method and around 4 min for the integral equation method on a PC with $2.6 \mathrm{GHz}$ CPU.

Finally, we consider a 3D reservoir model with bathymetry. The bathymetry is exactly the same as in the previous model. The anomalous domain for the resistive $\mathrm{HC}$ reservoir is defined as follows: $\Omega_{a}=\{-500 \mathrm{~m} \leq x, y \leq 500 \mathrm{~m} ; 2000 \mathrm{~m} \leq z \leq 2100 \mathrm{~m}\}$. We also
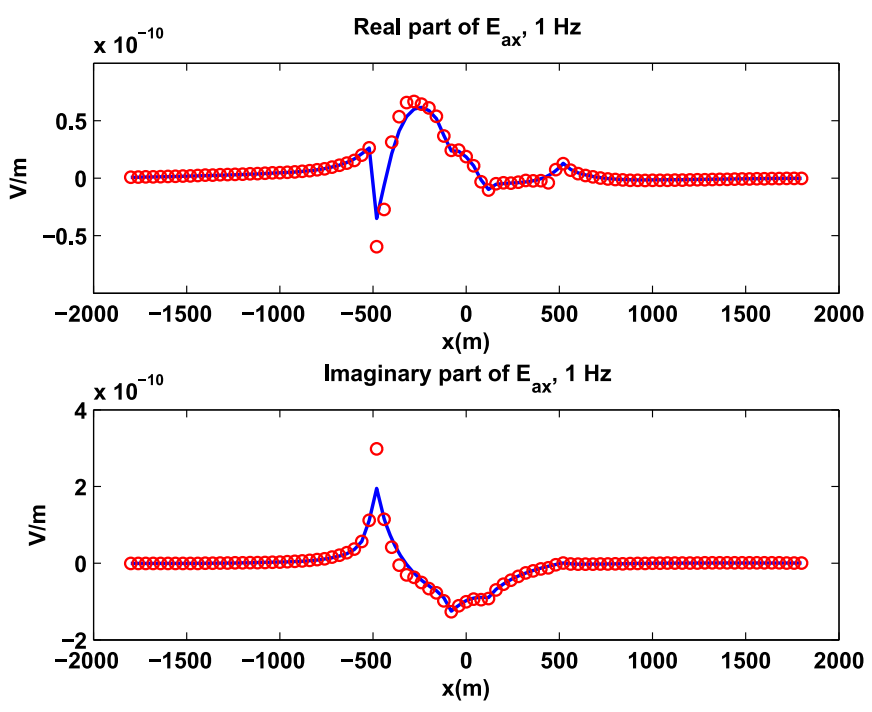

Fig. 28. Bathymetry model with the HC reservoir. A comparison of the $x$ component of the anomalous electric field computed using the finite element (red circles) and integral equation (blue line) methods at $y=0$ along the sea floor for a frequency of $1 \mathrm{~Hz}$. (For interpretation of the references to color in this figure caption, the reader is referred to the web version of this paper.) 


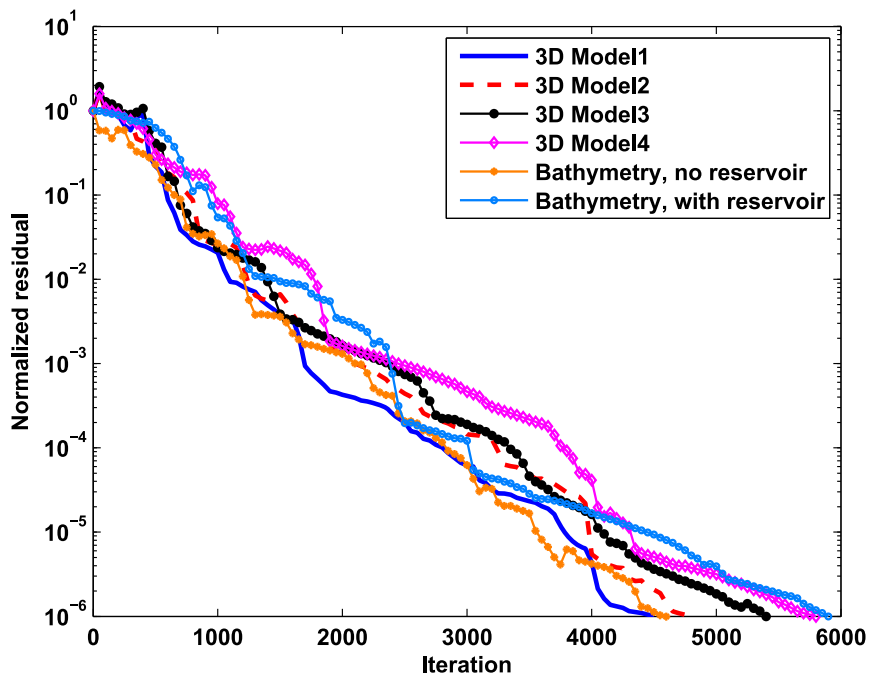

Fig. 29. Comparison of QMR convergence plots for different 3D reservoir models presented in this paper.

consider anisotropic conductivities of the sediment and the reservoir. The horizontal and vertical conductivities of the sediment are equal to $1 \mathrm{Sm}^{-1}$ and $0.8 \mathrm{Sm}^{-1}$, respectively. The horizontal and vertical conductivities of the HC reservoir are selected as follows: $0.05 \mathrm{Sm}^{-1}$ and $0.005 \mathrm{Sm}^{-1}$. Fig. 27 shows the hexahedral grid for the bathymetry model with the HC reservoir in the $X-Z$ section at $y=0$. Fig. 28 presents the plots of the anomalous electric field computed using edge-based finite element and integral equation methods at $y=0$ on the seafloor with the frequency of $1 \mathrm{~Hz}$. One can see that the results produced by these two methods show a good agreement. Some minor difference may be related to the staircase approximation used in the integral equation method.

For this bathymetry model with a reservoir, the total memory required to solve this model using finite element and integral equation methods are $1.8 \mathrm{~GB}$ and $300 \mathrm{MB}$, respectively. It took about 32 min to solve this model using the finite element method and around $5 \mathrm{~min}$ for the integral equation method on a PC with $2.6 \mathrm{GHz} \mathrm{CPU}$.

At the end, we present the convergence plots of QMR solvers for different 3D reservoir models, that we tested in this paper, in Fig. 29. We can see that the normalized residuals decrease steadily with the iteration number even in the presence of submarine topography and conductivity anisotropy.

\section{Conclusions}

We have developed an edge-based finite element algorithm to solve the diffusive electromagnetic problem in the 3D anisotropic medium. This method can be specifically useful for modeling geophysical electromagnetic data observed by marine controlledsource electromagnetic (MCSEM) surveys in the areas with anisotropic conductivity of the sea-bottom geological formations and a complex bathymetry. We have considered a typical MCSEM survey with an electric dipole source. In order to avoid the source singularity, we solve Maxwell's equations with respect to the anomalous electric field. We use the edge-based vector basis functions, which automatically enforce the divergence free conditions for electric and magnetic fields. Moreover, the continuity of tangential electric and magnetic fields is satisfied automatically as well. The sparse finite element system is solved using the quasiminimum residual method with a Jacobian preconditioner.
The developed code was tested for a number of typical geoelectrical models of the off-shore HC reservoir. The results of numerical study confirm the accuracy and the efficiency of a new code. Future work will be aimed at the implementation of the high order finite elements and at the use of the unstructured tetrahedral and hexahedron meshes to include seafloor bathymetry and complex geoelectrical structures in the modeling of the MCSEM data.

\section{Acknowledgement}

The authors acknowledge the support of the University of Utah's Consortium for Electromagnetic Modeling and Inversion (CEMI) and TechnoImaging. Thanks to Dr. Gribenko for his suggestions and fruitful discussions. Thanks to our CEMI student Daeung Yoon for creating finite difference sparsity matrix presented in this paper. The project was partially supported by the National Natural Science Foundation of China with Grant codes of 40974077 and 41164004. The authors are thankful to both reviewers for their valuable comments and suggestions which helped to improve the paper.

\section{References}

Anderson, W.L., 1989. A hybrid fast Hankel transform algorithm for electromagnetic modeling. Geophysics 54, 263-266.

Axelsson, O., 1994. Iterative Solution Methods. Cambridge University Press, New York.

Andréis, D., MacGregor, L., 2008. Controlled-source electromagnetic sounding in shallow water: principles and applications. Geophysics 73, F21-F32.

Brown, V., Hoversten, M., Key, K., Chen, J., 2012. Resolution of reservoir scale electrical anisotropy from marine CSEM data. Geophysics 77, E147-E158.

Constable, S., Srnka, L.J., 2007. An introduction to marine controlled-source electromagnetic methods for hydrocarbon exploration. Geophysics 72 WA3-WA12.

Clavaud, J.B., 2008. Intrinsic electric anisotropy of shale: the effect of compaction. Petrophysics 49, 243-260.

Ellis, M., Sinha, M., Parr, R., 2010. Role of fine-scale layering and grain alignment in the electrical anisotropy of marine sediments. First Break 28, 49-57.

Freund, R.W., Nachtigal, N.M., 1991. QMR: a quasi-minimal residual method for non-Hermitian linear systems. Numer. Math. 60, 315-339.

Guptasarma, D., Singh, B., 1997. New digital linear filters for Hankel J0 and J1 transforms. Geophys. Prospect. 54, 263-266.

Jin, J., 2002. Finite Element Method in Electromagnetics. Wiley-IEEE Press, New York.

Kong, F.N., Johnstad, S.E., Rosten, T., Westerdahl, H., 2008. A 2.5D finite-elementmodeling difference method for marine CSEM modeling in stratified anisotropic media. Geophysics 73, F9-F19.

Løseth, L.O., Ursin, B., 2007. Electromagnetic fields in planarly layered anisotropic media. Geophys. J. Int. 170, 44-80.

Li, Y., Dai, S., 2011. Finite element modelling of marine controlled-source electromagnetic responses in two-dimensional dipping anisotropic conductivity structures. Geophys. J. Int. 185, 622-636.

Mitsuhata, Y., Uchida, T., 2004. 3D magnetotelluric modeling using the T- $\omega$ finiteelement method. Geophysics 69, 108-119.

Newman, G.A., Commer, M., Carazzone, J.J., 2010. Imaging CSEM data in the presence of electrical anisotropy. Geophysics 75, F51-F61.

Nédélec, J.C., 1980. Mixed finite elements in $\mathrm{R}^{3}$. Numer. Math. 35, 315-341.

Negi, J.G., Saraf, P.D., 1989. Anisotropy in Geoelectromagnetism. Elsevier, Amsterdam.

Puzyrev, V., Koldan, J., Puente, J., Houzeaux, G., Vázquez, M., Cela, J.M., 2013. A parallel finite-element method for three-dimensional controlled-source electromagnetic forward modelling. Geophys. J. Int. 193, 678-693.

Ramananjaona, C., MacGregor, L., Andréis, D., 2011. Sensitivity and inversion of marine electromagnetic data in a vertically anisotropic stratified earth. Geophys. Prospect. 110, 341-360.

Saad, Y, 2003. Iterative Methods for Sparse Linear Systems. SIAM, Philadelphia.

Srnka, L.J., Carazzone, J.J., Ephron, M.S., Eriksen, E.A., 2006. Remote reservoir resistivity mapping. Lead. Edge 25, 972-975.

Sasaki, Y., 2011. Bathymetric effects and corrections in marine CSEM data. Geophysics 76, F139-F146.

Schwarzbach, C., Börner, R.U., Spitzer, K., 2011. Three-dimensional adaptive higher order finite element simulation for geo-electromagnetics-a marine CSEM example. Geophys. J. Int. 187, 63-74. 
Silva, N.V., Morgan, J.V., MacGregor, L., Warner, M., 2012. A finite element multifrontal method for 3D CSEM modeling in the frequency domain. Geophysics 77, E101-E115.

Tompkins, M., 2005. The role of vertical anisotropy in interpreting marine controlled-source electromagnetic data. In: 75th Annual International Meeting, SEG, Expanded Abstract, pp. 514-517.

Um, E.S., Alumbaugh, D.L., 2007. On the physics of the marine controlled-source electromagnetic method. Geophysics 72, WA13-WA26.

Van der Vorst, H.A., 1992. Bi-CGSTAB: a fast and smoothly converging variant of BiCG for the solution of nonsymmetric linear systems. SIAM J. Sci. Stat. Comput. 13, 631-644.

Van der Vorst, H.A., 2003. Iterative Krylov Methods for Large Linear Systems. Cambridge University Press, New York.

Ward, S.H., Hohmann, G.W., 1988. Electromagnetic Theory for Geophysical Applications. SEG, Oklahoma.
Weiss, C.J., Newman, G.A., 2002. Electromagnetic induction in a fully 3D anisotropic earth. Geophysics 67, 1104-1114.

Zhdanov, M.S., Keller, G., 1994. The Geoelectrical Methods in Geophysical Exploration. Elsevier, Amsterdam.

Zhdanov, M.S., Lee, S.K., Yoshioka, K., 2006. Integral equation method for 3D modeling of electromagnetic fields in complex structures with inhomogeneous background conductivity. Geophysics 71, 333-345.

Zhdanov, M.S., 2009. Geophysical Electromagnetic Theory and Methods. Elsevier, Amsterdam.

Zhdanov, M.S., 2010. Electromagnetic geophysics: notes from the past and the road ahead. Geophysics 75, A49-A66. 